\title{
Efficiently sphere-decodable physical layer transmission schemes for wireless storage networks
}

\author{
Hsiao-feng (Francis) Lu ${ }^{1}$, Amaro Barreal ${ }^{2}$, David Karpuk ${ }^{2}$ and Camilla Hollanti2 ${ }^{*}$
}

\begin{abstract}
Three transmission schemes over a new type of multiple-access channel (MAC) model with inter-source communication links are proposed and investigated in this paper. This new channel model is well motivated by, e.g., wireless distributed storage networks, where communication to repair a lost node takes place from helper nodes to a repairing node over a wireless channel. Since in many wireless networks nodes can come and go in an arbitrary manner, there must be an inherent capability of inter-node communication between every pair of nodes. Assuming that communication is possible between every pair of helper nodes, the newly proposed schemes are based on various smart time-sharing and relaying strategies. In other words, certain helper nodes will be regarded as relays, thereby converting the conventional uncooperative multiple-access channel to a multiple-access relay channel (MARC). The diversity-multiplexing gain tradeoff (DMT) of the system together with efficient sphere-decodability and low structural complexity in terms of the number of antennas required at each end is used as the main design objectives. While the optimal DMT for the new channel model is fully open, it is shown that the proposed schemes outperform the DMT of the simple time-sharing protocol and, in some cases, even the optimal uncooperative MAC DMT.

While using a wireless distributed storage network as a motivating example throughout the paper, the MAC transmission techniques proposed here are completely general and as such applicable to any MAC communication with inter-source communication links.
\end{abstract}

Keywords: Distributed communications, Distributed storage systems, Diversity-multiplexing gain tradeoff, MIMO, Multiple-access channel, Relay channel, Sphere decoding, Wireless networks

\section{Introduction}

The amount of data in cloud storage systems and worldwide data traffic have reached incredible numbers. It was estimated that in $2011,1.8 \cdot 10^{21}$ bytes of data needed to be stored worldwide [1], a number that grew to an astonishing $4.4 \cdot 10^{21}$ bytes in 2013 , and which is further expected to grow tenfold by 2020 [2]. The availability of such an astronomical amount of data and rapid progress in (wireless) communications engineering explain the observed growth of mobile data traffic, which increased by $69 \%$ in 2014, reaching $2.5 \cdot 10^{18}$ bytes per month at the end of

${ }^{*}$ Correspondence: camilla.hollanti@aalto.fi

${ }^{2}$ Department of Mathematics and Systems Analysis, Aalto University, P.O. Box

11100, FI-00076 AALTO (Espoo), Finland

Full list of author information is available at the end of the article the year. This amount of data traffic, which is expected to increase tenfold until 2019, corresponds to nearly 30 times the size of the entire internet in 2000. In addition, about 497 million mobile devices and connections were added globally in 2014, of which smart phones account for $88 \%$, so that-as foreseen-the number of mobile-connected devices exceeded the number of people on earth by the end of 2014 [3].

The massive amount of available data demands that data no longer be stored on a single device, but rather distributed among several storage nodes in a network, hence usually referred to as distributed storage systems (DSSs) (see [4] for a nice introduction). One of the main advantages of storing information in a distributed manner is that the storage system can be made robust against failures by introducing some level of redundancy. Some examples of 
real-life distributed storage systems are Apache Cassandra [5], which is a DSS initially developed at Facebook, and Windows Azure [6], created by Microsoft.

More formally, a DSS consists of $n$ storage nodes over which a file is stored in a redundant manner by dividing it into fragments and distributing the fragments among $n$ nodes using, for instance, a $(n, k)$ maximum distance separable (MDS) erasure code [7]. MDS codes satisfy the Singleton bound and have the convenient property of being able to reconstruct the file by contacting any $k$ of the nodes. Another key feature of a DSS is the ability to repair, meaning that when a node fails (that is a device breaks down or leaves the network), the failed node can be repaired or replaced. If an $(n, k)$ storage code further satisfies the condition that any failed node can be repaired or replaced by contacting any $K$ of the remaining nodes, termed helpers, the code is called an $(n, k, K)$ storage code, and the node replacing the failed one is called a repairing node, or a newcomer [8], if it is not one of the already existing nodes in the network.

Sophisticated storage protocols have been developed, always giving a tradeoff between the amount of data that needs to be stored in any of the storage nodes, and the amount of data that needs to be retrieved for repairing a lost node, also called repair bandwidth (see e.g. $[8,9]$. for details), and codes lying on the storage-repair bandwidth tradeoff curve $[8,10]$ are called regenerating codes. Explicit, tradeoff achieving regenerating codes can be found in the literature, see $[9,11]$, among others.

One important aspect of future DSSs lies in the ability to communicate over wireless channels, making it possible to store or retrieve a file using a wireless connection, even if the storage cloud itself might be wired. This is a feature related to the more general concept of wireless edge [12-15]. The mobility of a user has become crucial in everyday life, and wireless channels are used for data transmission for increased flexibility. However, it is wellknown that communicating over a fading channel in a wireless DSS $[16,17]$ makes repair transmissions prone to physical layer errors.

Consider the wireless repair transmission of a DSS, that is, the case of repairing a failed/lost node and replacing it with a repairing node by contacting any $K$ of remaining storage (helper) nodes via wireless links. We assume that both types of nodes may be equipped with multiple antennas. Then, the transmission from the $K$ helpers to the repairing node can be regarded as wireless multiple-input multiple-output (MIMO) multiple-access communication [18-20] with an additional feature of inter-helper communication among the $K$ helpers. To see this, note that the MIMO multiple-access channel (MAC) studied in classical information theory [21] assumes only the existence of communication links from the helpers to the repairing node, or equivalently from sources to destination. Yet in many wireless distributed storage networks ${ }^{1}$, there are often more nodes present and connected than those storing data, i.e., there are blank nodes in addition to the actual storage nodes. The total number of nodes can be dynamic even though the number of storage nodes would be fixed, see Fig. 1 for illustration. Now, the loss of a node can happen to any of the $n$ storage nodes, and the $K$ helpers can be any subset of the remaining storage nodes. The role of a repairing node can be taken by any of the blank nodes (also a new node entering the system will be blank in the beginning). This implies that an inherent communication link exists between the blank nodes and the storage nodes. After repair, a node that was previously blank becomes a storage node, and after this can assume the role of a helper node. Storage nodes may also erase their stored data and become blank, after which they can also assume the role of a repairing node, etc. This means that an inter-node communication capability actually exists between every pair of helper nodes, therefore calling for the design of efficient transmission schemes when the sources are further allowed to communicate with each other in a wireless MIMO-MAC.

Yet another example to motivate such inter-helper links is as follows. Notice that each of the $n$ wireless storage nodes in the network consists of a wireless component and a memory component. In case of the wireless component of a node failing, we simply replace it with a new wireless component, and there is no need to contact the helpers to reconstruct the data. On the other hand, if the memory component fails, the "repair" of the node (hence the name of repairing node) happens by repairing the memory component of the node with the aid of helper nodes. That is, the node is not replaced by a completely new node. In this sense, the failed node is still one of the original $n$ storage nodes, and the repairing process is done by contacting any $K$ of the remaining nodes. In other words, the failed node and the repairing node are the same node with the same wireless component. This justifies the requirement that the inter-helper link must exist between every pair of nodes.

When communicating over a wireless channel between terminals equipped with multiple antennas, space-time codes [22-26] are often employed to protect the transmitted information from adverse channel effects such as fading and noise. The asymptotic error-performance of space-time codes is commonly dictated by the diversitymultiplexing gain tradeoff (DMT) [27]. Assume each of the $K$ helper nodes has $n_{t}$ transmit antennas and transmits simultaneously at the same rate of $R=r \log _{2}$ SNR in bits per channel use to the repairing node with $n_{r}$ receive antennas, where SNR is the signal-to-noise power ratio, and $r$ is commonly referred to as the multiplexing gain [27]. The optimal MIMO-MAC DMT was given by Tse et al. in [28] and characterizes the maximal diversity gain, 


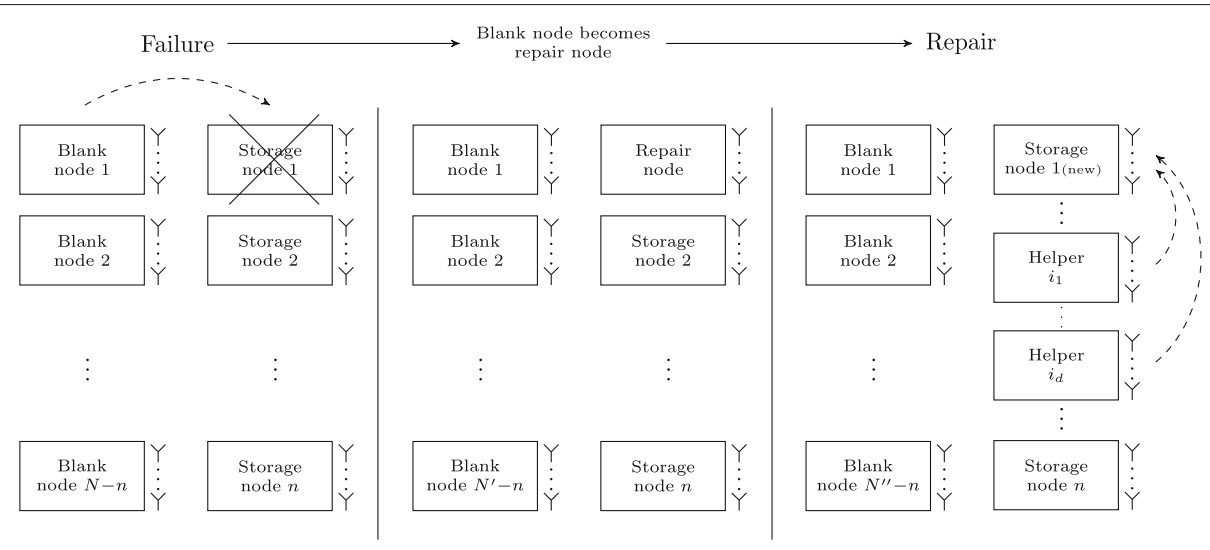

Fig. 1 Wireless storage network. A dynamic network with a varying number of nodes, out of which a fixed number of $n$ nodes are storing data and the rest are inactive (blank)

or equivalently the smallest error probability, that can possibly be achieved by any space-time code. A general construction of MIMO-MAC DMT optimal space-time codes was proposed in [26] for any triple $\left(n_{t}, n_{r}, K\right)$ and multiplexing gain $r$. These MIMO-MAC codes are constructed from cyclic division algebras $[23,29]$ and have a lineardispersion form [30]. Therefore, they can be decoded in the maximal-likelihood (ML) sense by a sphere decoder [31]. While sphere decoding is known to be an efficient implementation of ML decoding, it is unfortunate that when $K n_{t}>n_{r}$, decoding these DMT-optimal codes requires at least partial brute-force decoding before the sphere decoder begins to function [32], or alternatively one has to allow for suboptimal decoding methods, incurring degraded performance [33]. The former approach, though achieving the optimal performance, results in an exponential increase of decoding complexity when $K n_{t}$ becomes large and $n_{t}$ remains fixed [32].

Motivated by these realistic problems in wireless DSSs, new transmission schemes based on various strategies are proposed in this paper. The DMT, together with efficient sphere-decodability and low transmitter and receiver structural complexity in terms of the number of antennas required at each end, are used as the main design objectives, thus naturally establishing a DMT-complexity tradeoff.

\subsection{Contributions and related work}

In most of the storage and network-coding related research the focus is on the (logical) network layer, while the physical layer functionality is usually ignored or assumed perfect. An exception is [16], where a so-called partial downloading scheme is proposed, which allows for data reconstruction with limited bandwidth by downloading only parts of the content of helper nodes. This is relevant in the fading channel scenario, and the idea can potentially be combined with the present work. In
[17], optimal storage codes are constructed for the errorand-erasure scenario, but fading is not addressed. Isolated from the storage point of view, a lot of research has been carried out in physical layer wireless communications, see e.g. [34] and the references therein.

Remark 1. An obvious, but naïve attempt would be to try to simply combine an optimal storage code on the network layer and an optimal space-time code on the physical layer. The reason for avoiding this approach is both its structural (many antennas) and computational (decoding subject to partial brute-force) complexity.

In this paper ${ }^{2}$, we propose a class of transmission schemes for MIMO-MAC when communication links among the sources (helper nodes) do exist, which is generally true in many wireless storage networks. The proposed schemes allow for the design of efficiently sphere-decodable space-time codes ${ }^{3}$ with only one or two receive antennas. This is in contrast to the state-of-the-art MIMO-MAC codes [26] that have extremely good performance but require $K n_{t}$ receive antennas at the repairing node to enable efficient sphere decoding. This is of course unacceptable even for a relatively small value of $K$, since wireless networks are often heterogenous and might include nodes with only few or even just one antenna. At the moment, to the best of the authors' knowledge, no such scheme exists for large value of $K$ when the receiver has only 1 or 2 antennas, except for the trivial scheme of time-sharing among $K$ helper nodes.

This paper is organized as follows. In Section 2, we will present the channel model for DSS repair transmission, which can be seen as a MIMO-MAC in the presence of communication links among helper nodes. A brief introduction on DMT will also be given therein. Section 2.2 briefly reviews the notion of complexity exponent, which was established by Jaldén and Elia [35] for measuring 
the minimal computational complexity required by sphere decoders in order to achieve a certain diversity performance. The complexity exponent of existing MIMOMAC DMT optimal codes [26, 32, 36] is also given to further motivate our design objectives that are presented in Section 3 and to serve as a baseline for comparing the complexity of the first proposed scheme given in Section 4 . The first scheme is based on a simple time sharing among pairs of helpers, when $n_{t}=1, n_{r}=2$ and $K \geq 2$. The resulting DMT falls between the simple time sharing DMT and optimal MIMO-MAC DMT [28].

We then present two more elaborate schemes extending the first one, achieving a higher DMT by taking advantage of the inter-helper communication links and transforming the overall DSS network into a series of relay networks, where conventional half-duplex ${ }^{4}$ cooperativecommunication protocols such as the non-orthogonal amplify-and-forward (NAF) strategy $[37,38]$ will be used. In particular, it will be seen that these schemes can outperform the MIMO-MAC DMT at certain multiplexing gains, simply due to the use of inter-helper communications in the DSS. Moreover, our results on the DMTs for NAF-based relay networks not only improve, but also extend the ones presented in related works, such as $[39,40]$.

\section{Transmission model and preliminaries}

Consider a wireless DSS with $K$ helper nodes, equipped with $n_{t}$ transmit antennas each, and a repairing node with $n_{r}$ receive antennas. Let $H_{i} \in \mathbb{C}^{n_{r} \times n_{t}}$ be the channel matrix, and $X_{i} \in \mathbb{C}^{n_{t} \times T}$ the code matrix associated with the $i$ th helper node, where $T$ is the number of channel uses needed for transmitting $X_{i}$. The received signal matrix at the repairing node is given by

$$
Y=\sum_{i=1}^{K} H_{i} X_{i}+W
$$

where $W \in \mathbb{C}^{n_{r} \times T}$ is a matrix modeling complex additive white Gaussian noise (AWGN). The entries of $H_{i}$ and $W_{i}$ are independent and identically distributed (i.i.d.) circularly symmetric complex Gaussian random variables with zero mean and unit variance, a distribution which we henceforth denote as $\mathbb{C N}(0,1)$. The code matrices $X_{i}$ are required to satisfy the average power constraint $\mathbb{E}\left\|X_{i}\right\|^{2} \leq$ $T$. SNR. It is also assumed throughout the paper that the repairing node has a complete knowledge of channel state information $\left\{H_{i}: i=1, \ldots, K\right\}$.

Due to the nature of the DSS, the helper nodes can communicate with each other, a feature not seen in classical MIMO-MAC. Focusing on the $i$ th helper node, let $G_{i, j} \in \mathbb{C}^{n_{t} \times n_{t}}$ be the channel matrix and $S_{j} \in \mathbb{C}^{n_{t} \times T^{\prime}}$ be the code matrix sent by the $j$ th helper node, $j \neq i$; then, the signal matrix received at the $i$ th helper node is given by

$$
Y_{i}=\sum_{\substack{j=1 \\ j \neq i}}^{K} G_{i, j} S_{j}+Z_{i},
$$

where the entries of $G_{i, j}$ and $Z_{i}$ are again modeled as i.i.d. $\mathbb{C N}(0,1)$ random variables, and the signal matrices $S_{j}$ satisfy $\mathbb{E}|| S_{j} \|^{2} \leq T^{\prime}$. SNR. A complete knowledge of $\left\{G_{i, j}\right\}$ is assumed to be available at the $i$ th node. Finally, it is assumed throughout the paper that all communication links are half-duplex. A pictorial description of the above channel model is given in Fig. 2.

\subsection{The DMT}

One of the design objectives in this paper is to provide high performance transmission schemes for wireless DSS repair transmissions. The performance of each scheme will be measured by the DMT $[27,34]$. In order to simplify the discussion of DMT, let us ignore the existence of the inter-helper channels for the moment and focus only on the channel input-output relation (1), where only the direct channels from the $K$ helper nodes to the repairing node are of concern. Assuming each helper node transmits at the same multiplexing gain $r$ to the repairing node, we say a scheme achieves diversity gain $d(r)$ if its outage probability $P_{\text {out }}(r)$, which is defined as the probability of mutual information $I\left(X_{i_{1}}, \ldots, X_{i_{s}} ; Y \mid H_{1}, \ldots, H_{K}\right)$ being strictly less than $s \cdot r \log _{2}$ SNR for some $\left\{i_{1}, \ldots, i_{s}\right\} \subseteq$ $\{1, \ldots, K\}$, satisfies

$$
-\lim _{\mathrm{SNR} \rightarrow \infty} \frac{\log P_{\text {out }}(r)}{\log \mathrm{SNR}}=d(r)
$$

and we will write the above as

$$
P_{\text {out }}(r) \doteq \mathrm{SNR}^{-d(r)} \text {. }
$$

The outage probability $P_{\text {out }}(r)$ is an asymptotic lower bound on the error probability of the scheme [27, 34]

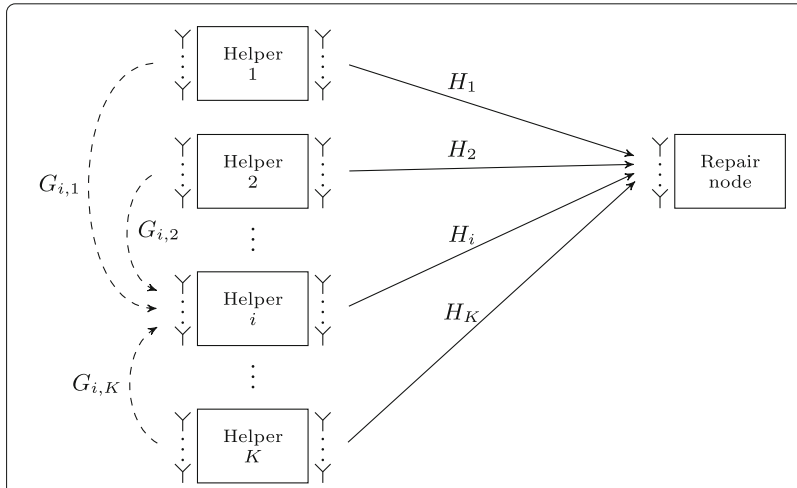

Fig. 2 DSS repair transmission. Complete channel model for DSS repair transmission with $K$ helper nodes, each having $n_{t}$ transmit antennas, and with $n_{r}$ receiver antennas at the repairing node 
when SNR is large and the multiplexing gain $r$ remains fixed.

For objectivity, we will compare the performance of the proposed schemes with the following optimal DMT [28] for MIMO-MAC ${ }^{5}$

$$
d_{n_{t}, n_{r}, K}^{*}(r)=\min \left\{d_{n_{t}, n_{r}}^{*}(r), d_{K n_{t}, n_{r}}^{*}(K r)\right\},
$$

where $d_{m, n}^{*}(r)$ is the optimal DMT for an $\left(n_{t} \times n_{r}\right)$ pointto-point MIMO channel and is given by the piecewise linear function connecting the points $\left(r,\left(n_{t}-r\right)\left(n_{r}-r\right)\right)$ for $r=0,1, \ldots, \min \left\{n_{t}, n_{r}\right\}$ [27]. We must emphasize that (5) is known to be optimal for the non-cooperative MIMO-MAC, that is, the case when the $K$ helper nodes share no common information, meaning that the interhelper channels (2) do not exist, and the file fragments stored at the $K$ helper nodes are all statistically independent $^{6}$. Therefore, with a properly designed scheme, it is expected that a higher DMT performance than (5) can be achieved in DSS repair transmission.

\subsection{Sphere decoding complexity of state-of-the-art MIMO-MAC codes}

A general construction of MIMO-MAC space-time codes was proposed in [26] and was shown to achieve the optimal DMT (5) for any triple $\left(n_{t}, n_{r}, K\right)$ and multiplexing gain $r$. More specifically, given $n_{t}$ and $K$, the component code $\mathcal{C}_{i}$ of the $i$ th helper node is taken from an algebraic lattice of lattice $\operatorname{rank}^{7} 2 n_{t} K_{o}^{2}$ in [26], and $\mathcal{C}_{i}$ consists of $\left(n_{t} \times T\right)$ matrices with $\left|\mathcal{C}_{i}\right| \doteq \mathrm{SNR}^{r T}$ and $T=n_{t} K_{o}$, where $K_{o}$ is the smallest odd integer $\geq K$.

To estimate the complexity of decoding the overall code $\mathcal{C}_{1} \times \cdots \times \mathcal{C}_{K}$ using a joint sphere decoder, we follow [35] by using the notion of complexity exponent as a complexity measure.

Definition 1 (Complexity exponent [35]). Given the multiplexing gain $r$, let $\mathcal{C}_{r, k}$ be a lattice code consisting of $\left(n_{t} \times T\right)$ codeword matrices with $\left|\mathcal{C}_{r, k}\right| \doteq S N R^{r T}, k=$ $1, \ldots, K$. Let $\mathcal{D}_{r}$ be a decoder for the overall code $\mathcal{C}_{r}=$ $\mathcal{C}_{r, 1} \times \cdots \times \mathcal{C}_{r, K}$, subject to a computational constraint $N_{\max }(r)$, in floating point operations (flops) per T channel uses, in the sense that after $N_{\max }(r)$ flops, the decoder $\mathcal{D}_{r}$ must simply terminate, potentially prematurely and before completing the task, thus declaring an error. We then say $\mathcal{D}_{r}$ achieves diversity order $d(r)$ with complexity exponent $c(r)$ if $\mathcal{D}_{r}$ achieves error probability $P_{e} \doteq S N R^{-d(r)}$ using at most $N_{\max }(r) \doteq S N R^{c(r)}(c f$. (3),(4)) flops of computational reserves.

The above definition means that in order to decode the code $\mathcal{C}_{r}$ using a joint sphere decoder, one does not have to decode every received signal matrix, especially when the communication channel is deeply faded. Instead, one can enforce a complexity constraint (also called a halting policy) at the sphere decoder, say at most $N_{\max }(r)$ flops of computational reserves. By choosing $N_{\max }(r)$ large enough such that the probability of any premature termination of the sphere decoder is asymptotically no larger than $\mathrm{SNR}^{-d_{n_{t}, n_{r}, K}^{*}(r)}$, the overall error probability at most $2 \cdot \mathrm{SNR}^{-d_{n_{t}, n_{r}, K}^{*}(r)}$, thereby achieving the same diversity $d_{n_{t}, n_{r}, K}^{*}(r)$.

It was shown in $[32,36]$ that the complexity exponent for decoding the DMT optimal code [26] is given by

$$
\begin{aligned}
c_{n_{t}, n_{r}, K}(r)= & K_{o} r\left(K n_{t}-n_{r}\right) \cdot \mathbf{1}\left(K n_{t}>n_{r}\right) \\
& +\sup _{\underline{\mu} \in \mathcal{B}(r)} K_{o} n_{t} \sum_{i=1}^{v}\left(\frac{r}{n_{t}}-\left(1-\mu_{i}\right)^{+}\right)^{+},
\end{aligned}
$$

where $\mathbf{1}(\cdot)$ is the usual indicator function, $v=$ $\min \left\{K n_{t}, n_{r}\right\},(x)^{+}:=\max \{x, 0\}$ and

$$
\begin{aligned}
& \mathcal{B}(r) \\
& =\left\{\begin{array}{l}
\underline{\mu}=\left[\begin{array}{ll}
\mu_{1} & \cdots \mu_{v}
\end{array}\right]^{\top} \in \mathbb{R}^{v}: \begin{array}{l}
\mu_{1} \geq \cdots \geq \mu_{v} \geq 0, \\
\sum_{i=1}^{v}\left(\left|K n_{t}-n_{r}\right|+2 i-1\right) \mu_{i} \leq d_{n_{t}, n_{r}, K}^{*}(r)
\end{array}
\end{array}\right\} .
\end{aligned}
$$

There is an intuitive explanation for the term $K_{o} r\left(K n_{t}-n_{r}\right)$ in (6) when $K n_{t}>n_{r}$. Recall that the component code $\mathcal{C}_{i}$ is taken from a certain subset of an algebraic lattice $\Lambda_{i}$ of rank $2 n_{t}^{2} K_{o}$. This means that each codeword matrix $X_{i}$ of $\mathcal{C}_{i}$ is of the form $X_{i}=\sum_{\ell=1}^{n_{t}^{2} K_{o}} x_{i, \ell} C_{i, \ell}$, where $\left\{C_{i, \ell}: \ell=1, \ldots, n_{t}^{2} K_{o}\right\}$ is a basis for $\Lambda_{i}$, and the $x_{i, \ell}$ are independent QAM symbols taken from a certain set $\mathcal{A} \subset \mathbb{Z}[l]$ of size $\mathrm{SNR}^{\frac{r}{n_{t}}}$, $\imath=\sqrt{-1}$. Thus, we can rewrite (1) as

$$
Y=\sum_{i=1}^{K} \sum_{\ell=1}^{n_{t}^{2} K_{o}} H_{i} C_{i, \ell} x_{i, \ell}+W,
$$

or equivalently in a vector form

$$
\underline{y}=H \underline{x}+\underline{w},
$$

where $\underline{x}=\left[x_{1,1}, \ldots x_{1, n_{t}^{2} K_{o}}, \ldots x_{K, n_{t}^{2} K_{o}}\right]^{\top}, \underline{y}$ is the vectorization of the matrix $Y$, and $H$ is the corresponding matrix of size $\left(n_{r} K_{o} n_{t} \times K n_{t}^{2} K_{o}\right)$ by (8). When decoding (9) using a sphere decoder, one first performs a QR-decomposition of the matrix $H$, say $H=Q R$. If $K n_{t}>n_{r}$, the matrix $R$ is no longer upper triangular; it is a trapezoidal matrix with

$$
K n_{t}^{2} K_{o}-n_{r} K_{o} n_{t}+1=K_{o} n_{t}\left(K n_{t}-n_{r}\right)+1
$$

nonzero entries in the bottom row. Hence, any sphere decoder for (9) must first resolve - perhaps by bruteforce - the $|\mathcal{A}|^{K_{o} n_{t}\left(K n_{t}-n_{r}\right)}=\mathrm{SNR}^{K_{o} r\left(K n_{t}-n_{r}\right)}$ ambiguities before processing the root of the sphere decoding tree. The number of ambiguities then forms the first term in (6).

Remark 2. A different definition of complexity exponent has appeared in [41], where Damen et al. studied the 
number of flops required by a sphere decoder to decode a fixed-rate space-time code at various finite SNR values. In particular, they defined the complexity exponent as the logarithm to base $m$ of the number of flops required by a sphere decoder to complete its task, where $m$ is the length of vector $\underline{x}$ defined in (9). Below we highlight some of the major differences between Damens' definition of complexity exponent and the one considered in this paper (cf. Definition 1).

- Damens' definition focuses on a code with a fixed rate, and Definition 1 concerns more with the theoretical asymptote at high $S N R$ regime when the rate scales linearly with $\log _{2} S N R$.

- Definition 1 considers the possibility of having a halting policy, while Damens' definition requires the sphere decoder to complete its task at all channel realizations.

Remark 3. In [42] Damen et al. proposed to decode (9) by using GDFE-MMSE preprocessing followed by the sphere decoder when $\mathrm{Kn}_{t}>n_{r}$, in hope of making the matrix $R$ upper-triangular and avoiding the need of resolving the ambiguities. However, it can be seen from [32, 36] that at high SNR regime the matrix $R$ - after MMSE-GDFE preprocessing - is ill-conditioned with $K_{o} n_{t}\left(K n_{t}-n_{r}\right)$ number of singular values arbitrarily close to zero. This also explains the appearance of the first term in (6).

On the other hand, when the code has a fixed rate and operates in the low or moderate SNR regime, the MMSEGDFE approach does offer a certain complexity reduction with a negligible performance loss, as the singular values of $R$ are numerically well-behaved in general. Other approaches for further complexity reductions under such premises are also available in the literature. For instance, Barbero and Thompson [43] proposed a fixed-complexity sphere decoder, where the number of candidates to be searched at the $i$-th level of sphere decoding tree is at most $n_{i}$, thereby yielding a constant complexity $\prod_{i} n_{i}$. Another way to reduce complexity is through the various orderings of singular values of $R$. A comprehensive study in this direction can be found for example in [44]. We shall emphasize that the complexity exponents simulated in [43, 44] are both based on Damens' definition [41] (cf. Remark 2) because of the aforementioned premises.

In Fig. 3, we plot the complexity exponents for the sphere decoding of MAC DMT optimal codes $\mathcal{C}_{r}$ [26] when $n_{t}=2, K=5$, and $n_{r}=2,10,100$, respectively. It can be seen that these codes can be efficiently decoded by sphere decoders only when $n_{r} \gg K n_{t}$. Such a requirement is often impossible in practice, particularly in heterogeneous storage networks, where nodes may have only a small number of antennas in use.

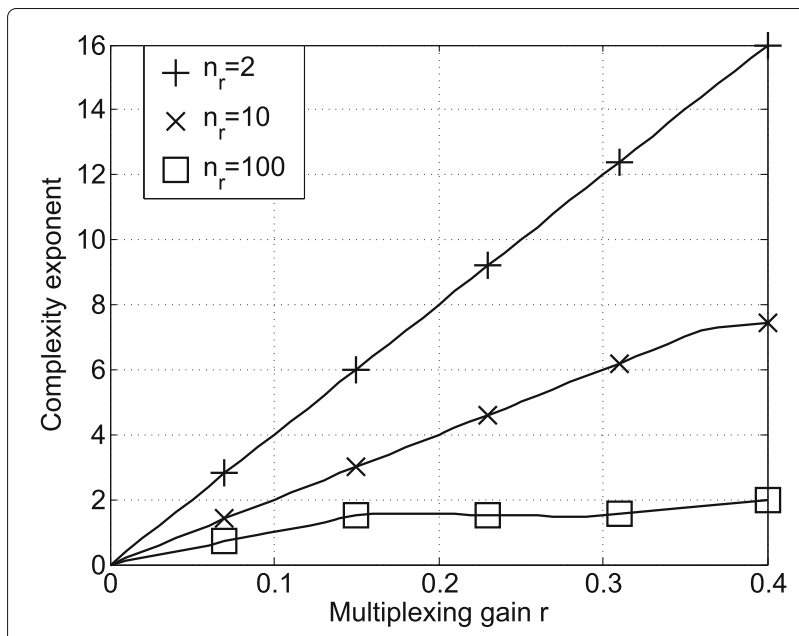

Fig. 3 Complexity exponent comparison. Complexity exponents for the sphere decoding of MAC-DMT optimal codes [26] when $n_{t}=2$, $K=5$ and $n_{r}=2,10,100$

Remark 4. In case of $n_{r} \geq K n_{t}$, it has been shown [45, 46] that the DMT optimal MIMO-MAC lattice codes can be decoded with sub-exponential complexity, i.e., having a complexity exponent asymptotically equal to 0 , using the Lenstra-Lenstra-Lovász-based lattice reduction aided regularized lattice decoder. The decoder is a combination of GDFE-MMSE, lattice reduction and sphere decoding, and it has a vanishing gap of performance loss to the exact $M L$ decoding as SNR approaches infinity.

\section{Objectives for the design of transmission schemes}

In Section 2, we have seen that there is a fundamental difference between the channel for DSS repair transmission and the classical MIMO-MAC, in the sense that the former includes additional inter-helper communication links. Thus, the MIMO-MAC DMT (5) and the MIMOMAC codes [26] are no longer optimal in scenarios such as DSS repair transmission. Moreover, due to these additional inter-helper channels, it is expected that the DSS repair transmission can have a higher optimal DMT than (5). This then calls for the design of new transmission schemes with good DMT performance for DSS repairing, which is the first design objective considered in this paper.

The second design objective comes from the observation of high decoding complexity of MIMO-MAC codes [26] in Fig. 3 when $K n_{t}>n_{r}$. In a DSS, it is often possible that $K$ is large, and $n_{r}$ is relatively small and fixed. This then calls for the design of new transmission schemes that can yield efficiently sphere-decodable spacetime codes avoiding the need to process the ambiguities by brute-force. Potentially, such an aim could be achieved by reducing the number of "active" helper nodes, i.e., reducing the effective value of $K$ in (1), such that the average 
number of independent QAM symbols received by the repairing node at each channel use be no larger than $n_{r}$, as observed from (6).

In the subsequent sections, we will focus on the case of $n_{t}=1$ and $K \gg n_{r}$, and we will provide three transmission schemes, each for a different configuration of the wireless DSS network and for a different design objective. The first scheme is given in Section 4 for the case of two receive antennas and an arbitrary number of helper nodes, each having one transmit antenna. It is based on a simple time sharing among pairs of helpers and is aimed at having a low sphere-decoding complexity at a cost of certain DMT performance-loss due to its neglect of existing inter-helper links. The DMT for this scheme falls between the simple time sharing DMT and optimal MIMO-MAC DMT (5).

Two more elaborate schemes will be presented in Sections 5 and 6, respectively, where we aim to improve the DMT performance at the possible $\operatorname{cost}^{8}$ of higher decoding complexity. These schemes take advantage of inter-helper channels and transform the overall DSS network into a series of relay networks, where the conventional half-duplex NAF protocol $[37,38]$ will be used. In particular, we will see that these schemes can outperform the MIMO-MAC DMT (5) at certain multiplexing gains, simply by exploiting inter-helper communications in the DSS.

\section{Scheme 1: $n_{t}=1, n_{r}=2$, and $K$ helper nodes}

We have seen in Section 2.2 that the existing state-ofthe-art MIMO-MAC space-time codes [26] could incur an extremely high decoding complexity when the repairing node has only a few number of antennas. Thus, our major aim in this section is to provide a new transmission scheme that can yield space-time codes with reduced decoding complexity. In particular, we would like these potential codes to be efficiently sphere-decodable, by which we mean that the $H$ matrix, when writing the channel input-output relation in a vector form (cf. (9)), has linearly independent columns with probability one.

Besides the desired property of being efficiently spheredecodable, the complexity of the transmission schemes should also be considered. In other words, if we ignore the existence of inter-helper links (2), then the schemes for DSS repair transmission can be made relatively simple. These are the main objectives of Scheme 1.

Let $\mathcal{K}=\{1,2, \ldots, K\}$ denote the set of $K$ helper nodes, and let $\mathcal{U}$ be a collection of two subsets sof $\mathcal{K}$, defined as below

$\mathcal{U}:= \begin{cases}\{\{1,2\},\{3,4,\}, \ldots,\{K-1, K\}\}, & \text { if } K \text { even, } \\ \{\{1,2\}, \ldots,\{K-2, K-1\},\{K, 1\},\{2,3\}, \ldots,\{K-1, K\}\}, & \text { if } K \text { odd. }\end{cases}$

With the above, the proposed scheme is the following. For each $U=\left\{u_{1}, u_{2}\right\} \in \mathcal{U}$, only helper nodes $u_{1}$ and $u_{2}$ are allowed to transmit during the active period of $U$. This implies that the probability of helper node $k$ transmitting equals $\frac{2}{K}$ for every $k \in \mathcal{K}$. In order to achieve an average multiplexing gain $r$, each helper node $k$, when chosen according to $U$, i.e. $k \in U$, should actually transmit at a higher multiplexing gain $\frac{K r}{2}$. We summarize the above scheme below, and a pictorial description of Scheme 1 is given in Fig. 4.
Scheme 1
1: for each $U=\left\{u_{1}, u_{2}\right\} \in \mathcal{U}$ do
2: Helper-nodes $u_{1}$ and $u_{2}$ transmit using the MIMO-MAC code given in [26, Eq. (20)] for $n_{t}=1$, two users and multiplexing gain $\frac{K r}{2}$.
3: end for

The following theorem is a straightforward consequence of [34].

Theorem 1. The DMT performance achieved by Scheme 1 is

$$
d_{1}(r)=\min \left\{d_{1,2}^{*}\left(\frac{K r}{2}\right), d_{2,2}^{*}(K r)\right\}
$$

In Fig. 5, we consider the case $n_{t}=1, n_{r}=2$ and $K=10$, and compare $d_{1}(r)$ to $d_{1,2,10}^{*}(r)$, which is the DMT corresponding to all 10 helper nodes transmitting simultaneously. The function $d_{0}(r)$ is the DMT for the time-division multiple-access (TDMA)-based scheme, by which we mean that each helper node takes turns in an orthogonal manner to transmit information to the repairing node at multiplexing gain $K r$. It can be seen that the first proposed scheme outperforms the TDMA scheme in terms of the DMT, and there is a considerable gap between $d_{1}(r)$ and $d_{1,2,10}^{*}(r)$. However, the comparison is unfair in the sense that in order to achieve $d_{1,2,10}^{*}(r)$ the codes in [26] would require exponentially large computational reserves, or equivalently an exponentially long time, for decoding.

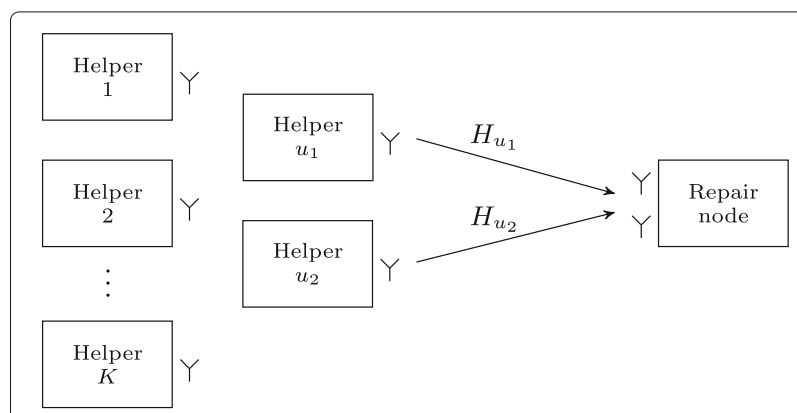

Fig. 4 Channel model of Scheme 1. Channel model for Scheme 1 at the Uth step, $U=\left\{u_{1}, u_{2}\right\} \subset\{1,2, \ldots, K\}$ 


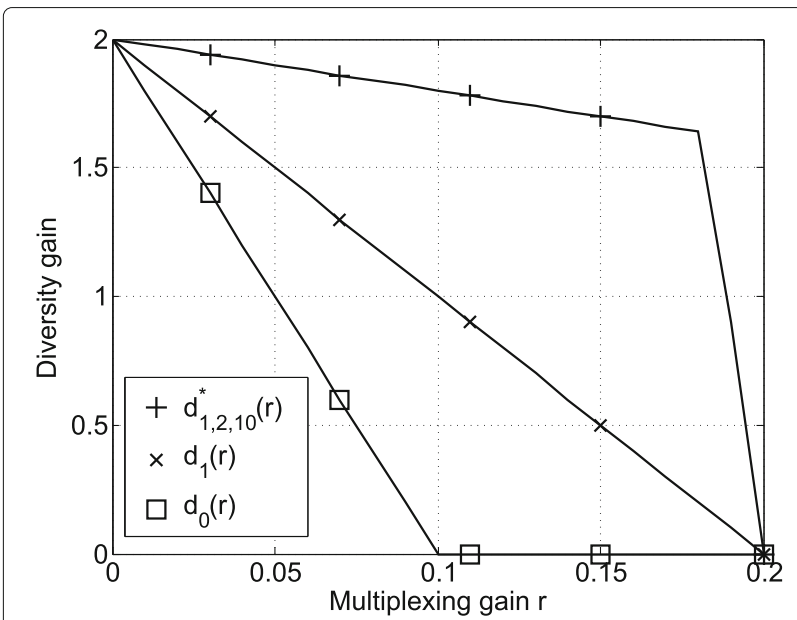

Fig. 5 Scheme 1: DMT comparison. DMT performances achieved by MIMO-MAC, Scheme 1 and time-sharing scheme for $K=10$ helper nodes, $n_{t}=1$, and $n_{r}=2$

Continuing the example of $n_{t}=1, n_{r}=2$ and $K=$ 10 , by modifying the two-user MIMO-MAC code given in [26] according to Scheme 1, it can be directly seen from (6) that the resulting code achieves DMT $d_{1}(r)$ with complexity exponent

$$
c_{1}(r):=3 \sup _{\underline{\mu} \in \mathcal{B}_{1}(r)} \sum_{i=1}^{2}\left[\min \left\{\frac{r K}{2}, \frac{K r}{2}+\mu_{i}-1\right\}\right]^{+},
$$

where

$\mathcal{B}_{1}(r)=\left\{\underline{\mu}=\left[\mu_{1} \mu_{2}\right]^{\top} \in \mathbb{R}^{2}: \mu_{1} \geq \mu_{2} \geq 0, \mu_{1}+3 \mu_{2} \leq d_{1}(r)\right\}$,

when it is decoded using a sphere decoder with halting policies. In Fig. 6, we compare $c_{1}(r)$ to the complexity exponent $c_{1,2,10}(r)$ of the MIMO-MAC code given in [26] for the case $n_{t}=1, n_{r}=2$ and $K=10$. It can be clearly seen that the proposed scheme can yield a code with with a much lower decoding complexity.

\section{Scheme 2: $n_{t}=1$, general $n_{r}$, and $K$ helper nodes}

The aim of Scheme 1 presented in the previous section was to have a small decoding complexity, at a cost of certain DMT performance loss due to the neglect of interhelper links in DSS repair-communication. In this section as well as the next, we will shift our focus to designing transmission schemes that take into account these interhelper links and beat the DMT performance $d_{n_{t}, n_{r}, K}^{*}(r)$.

Consider a DSS repair channel with $K$ helpers, each having $n_{t}=1$ transmit antenna, and a repairing node with $n_{r}$ receive antennas. To make good use of the inter-helper links, we interpret in Scheme 2 some of the links as links

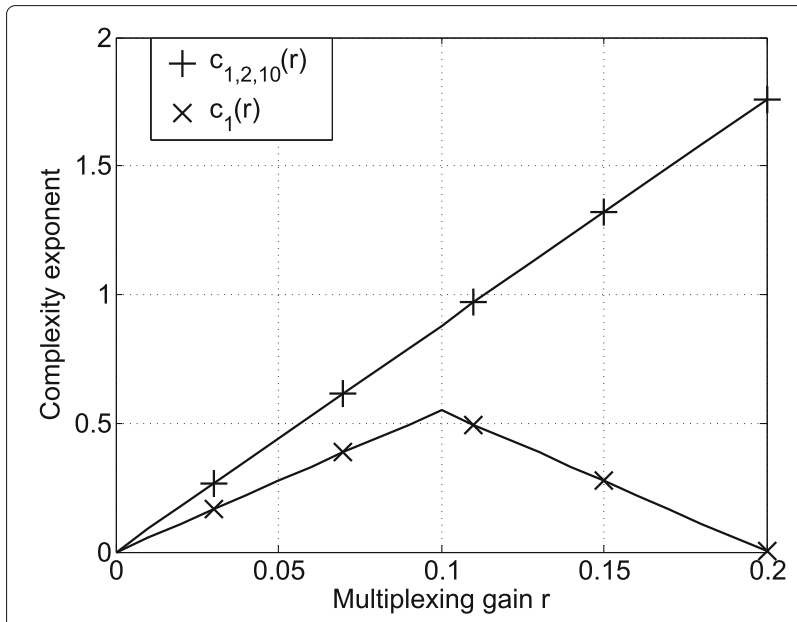

Fig. 6 Complexity exponent comparison. Complexity exponents for the sphere decoding of the MIMO-MAC code given in [26] $\left(c_{1,2,10}(r)\right)$ and the proposed code $\left(c_{1}(r)\right)$ based on Scheme 1 for the case of $n_{t}=1, n_{r}=2$ and $K=10$

of a relay channel. More specifically, in this scheme each of the $K$ helper nodes will take turns acting as the source in a cooperative relay network [37], while the remaining $K-1$ helper nodes play the role of relays helping the source to send information to the repairing node.

With the above, the proposed scheme is a modification of the NAF protocol $[37,38]$ for a cooperative relay network with $K-1$ relays. It consists of $K$ phases, and each phase requires at least $2(K-1)$ channel uses. Thus, the total number of channel uses required by Scheme 2 is at least $2 K(K-1)$.

Let $\mathcal{K}=\{1,2, \ldots, K\}$ denote the set of $K$ helper nodes. Given $k \in \mathcal{K}$, the scheme is at the $k$ th phase, and helper node $k$ acts as the source of a relay network. The remaining helper nodes $\mathcal{R}_{k}:=\mathcal{K} \backslash\{k\}=\left\{u_{1}, \ldots, u_{K-1}\right\}$ are the relays. At the $t$ th channel use of the $k$ th phase, $t=$ $1,2, \ldots, 2(K-1)$, node $k$ broadcasts a signal $x_{k, t}$, subject to the power constraint $\mathbb{E}\left|x_{k, t}\right|^{2} \leq \mathrm{SNR}$, to all nodes in $\mathcal{R}_{k}$ as well as to the repairing node. Due to the halfduplex assumption in Section 2, the nodes in $\mathcal{R}_{k}$ can either receive or transmit, but not both at the same time. Therefore, the behavior of each node $u_{i} \in \mathcal{R}_{k}$ is set such that it receives the signal from node $k$ when $t=2 i-1$ and transmits to the repairing node when $t=2 i$. More specifically, the signal received by node $u_{i}$ at $t=2 i-1$ is given by

$$
r_{u_{i}, 2 i-1}=g_{u_{i}, k} x_{k, 2 i-1}+z_{u_{i}, k, 2 i-1}
$$

where $g_{u_{i}, k}$ and $z_{u_{i}, k, 2 i-1}$ are i.i.d. $\mathbb{C N}(0,1)$ random variables representing the channel gain from node $k$ to node $u_{i}$ and the additive noise, respectively, as defined in (2). Node 
$u_{i}$ then amplifies the signal $r_{u_{i}, 2 i-1}$ with an amplification factor $a_{u_{i}, k}$ set such that

$$
\mathbb{E}\left|a_{u_{i}, k} r_{u_{i}, 2 i-1}\right|^{2} \leq \mathrm{SNR},
$$

where the expectation is taken with respect to $x_{k, 2 i-1}$ and $z_{u_{i}, k, 2 i-1}$, since $g_{u_{i}, k}$ is already known to node $u_{i}$. Equivalently, we have

$$
\left|a_{u_{i}, k}\right|^{2} \leq \frac{\mathrm{SNR}}{1+\mathrm{SNR}\left|g_{u_{i}, k}\right|^{2}} .
$$

Then, at channel use $t=2 i$, node $u_{i}$ joins node $k$ and sends the amplified signal $a_{u_{i}, k} r_{u_{i}, 2 i-1}$ to the repairing node.

Since each helper node $k$ is allowed to transmit its own message to the repairing node during the $k$ th phase, its multiplexing gain must be increased to $K \cdot r$ in order to achieve the desired average multiplexing gain $r$. We now summarize the steps of Scheme 2 below. A pictorial description of Scheme 2 is given in Fig. 7.

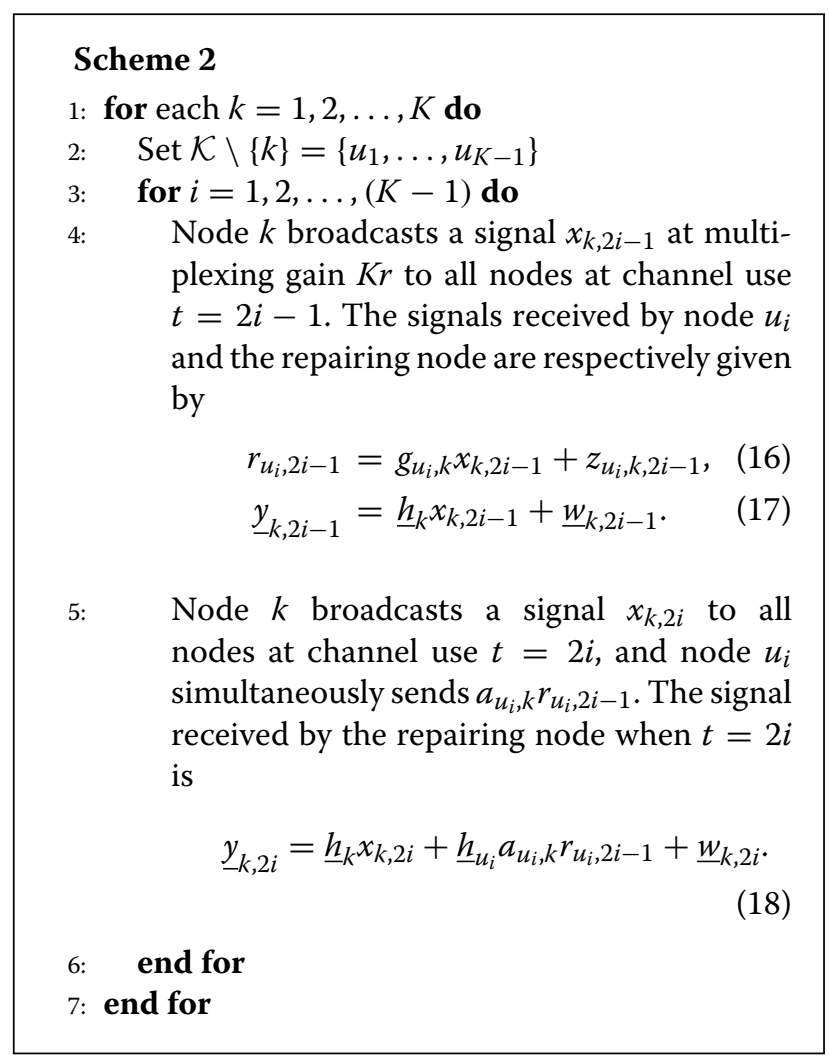

\subsection{DMT achieved by Scheme 2}

Note firstly that by the symmetry among the phases of Scheme 2, it suffices to analyze the DMT achieved within the first phase, i.e., for $k=1$, where the helper node 1 acts as the source, and the remaining helper nodes are relays. Thus, for notational convenience, we will henceforth drop the subindex $k$.
Set $N=2(K-1)$, and let $x_{t}$ be a $\mathbb{C N}(0, \mathrm{SNR})$ random variable, representing the signal sent by helper node 1 at time instance $t$ for $t=1,2, \ldots, N$. Then, the signal received by the repairing node at the $t$ th channel use is

$\underline{y}_{t}= \begin{cases}\underline{h}_{1} x_{t}+\underline{w}_{t}, & t \text { odd } \\ \underline{h}_{1} x_{t}+a_{i} \underline{h}_{i}\left(g_{i} x_{t-1}+z_{i}\right)+\underline{w}_{t}, & t \text { even and } i=\frac{t}{2}+1\end{cases}$

where $g_{i}$ and $z_{i}$ s are i.i.d. $\mathbb{C N}(0,1)$ random variables obtained by re-indexing the corresponding variables in (16) for notational convenience. The amplification factor $a_{i} \in \mathbb{R}^{+}, i=2, \ldots, K$, is set such that

$$
\left|a_{i}\right|^{2} \leq \frac{\mathrm{SNR}}{1+\mathrm{SNR}\left|g_{i}\right|^{2}} .
$$

We can equivalently reformulate the received vectors $\underline{y}_{t}$ in (19) in matrix form, as

$$
\underline{y}=\left[\begin{array}{llll}
\underline{y}_{1} & \underline{y}_{2} & \cdots & \underline{y}_{N}
\end{array}\right]^{\top}
$$

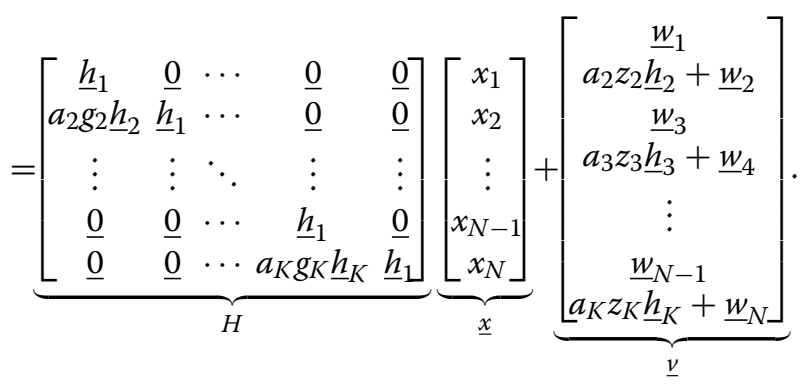

Given $H$, the instantaneous mutual information between the transmitted signal $\underline{x}$ and the received signal $\underline{y}$ is

$$
\begin{aligned}
I(\underline{x} ; \underline{y} \mid H) & =\log _{2} \operatorname{det}\left(K_{v}+\mathrm{SNR} H H^{\dagger}\right)-\log _{2} \operatorname{det}\left(K_{v}\right) \\
& =\sum_{i=2}^{K} \log _{2} \operatorname{det}\left(I_{2}+\mathrm{SNR} H_{i}^{\dagger} K_{i}^{-1} H_{i}\right),
\end{aligned}
$$

where

$K_{\nu}=\mathbb{E}\left(\underline{v} \underline{v}^{\dagger}\right), H_{i}=\left[\begin{array}{cc}\underline{h}_{1} & 0 \\ a_{i} g_{i} \underline{h}_{i} & \underline{h}_{1}\end{array}\right]$, and $K_{i}=\left[\begin{array}{cc}I_{n_{r}} & \\ & I_{n_{r}}+\left|a_{i}\right|^{2} \underline{h}_{i} \underline{h}_{i}^{\dagger}\end{array}\right]$.

Thus, the outage probability for Scheme 2 is given by

$\operatorname{Pr}\left\{H: \sup _{\left|a_{i}\right|^{2} \leq \frac{\mathrm{SNR}}{1+\mathrm{SNR}\left|\mathrm{g}_{i}\right|^{2}}} I(\underline{x} ; \underline{\underline{y}} \mid H)<2 K(K-1) r \log _{2} \mathrm{SNR}\right\} \doteq \mathrm{SNR}^{-d_{2}(r)}$,

where the target information rate $2 K(K-1) r \log _{2}$ SNR arises from the facts that

(i) the scheme takes $K$ phases to complete, and

(ii) each phase requires $2(K-1)$ channel uses. 


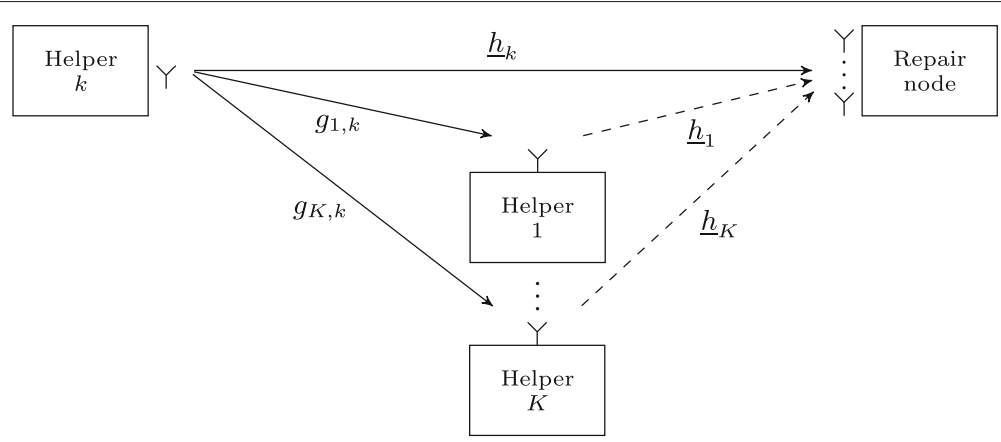

Fig. 7 Channel model of Scheme 2. Channel model for Scheme 2 at $k$ th phase

5.2 DMT achieved by Scheme 2 when $n_{r}=1$

When $n_{r}=1$, it can be seen that the DMT achieved by Scheme 2 is exactly the DMT for the NAF protocol derived by Azarian et al. [38] with $K-1$ relays and multiplexing gain $K r$. Hence, the following result is immediate from [38].

Theorem 2. The DMT achieved by Scheme 2 when $n_{r}=$ 1 is the following

$$
\left.d_{2}(r)\right|_{n_{r}=1}=(1-K r)^{+}+(K-1)(1-2 K r)^{+}
$$

In Fig. 8, we plot the DMT performance achieved by this scheme for the case of $K=10$ helper nodes. We also include the base-line TDMA scheme for comparison. It can be seen that the proposed scheme has a better DMT performance than $d_{n_{t}, n_{r}, K}^{*}(r)$ for $r \leq \frac{1}{2 K+1}=\frac{1}{21}$, due to the use of additional inter-helper links.

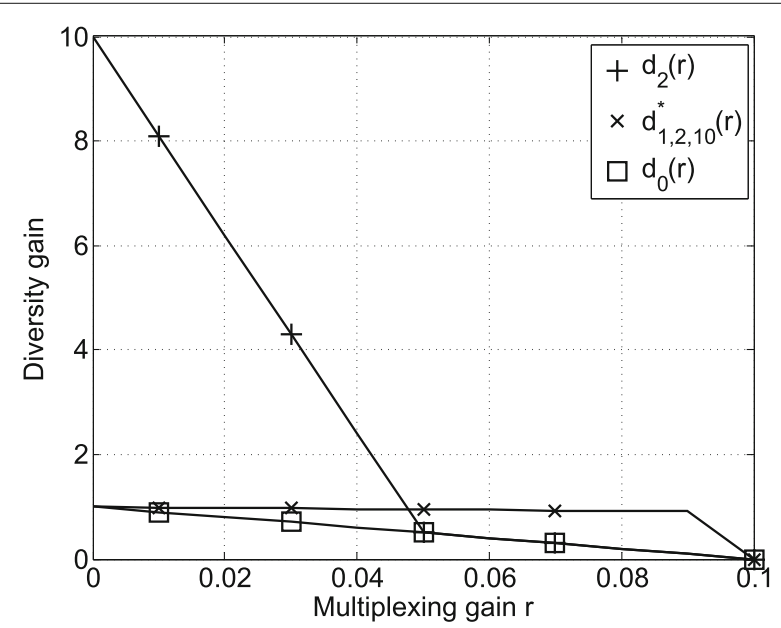

Fig. 8 Scheme 2: DMT comparison. DMT performances achieved by Scheme 2, MIMO-MAC and time-sharing scheme for $K=10$ helper nodes, $n_{t}=1$, and $n_{r}=1$
5.3 Upper and lower bounds on $d_{2}(r)$ with general $n_{r}$

Analyzing the outage probability (23) turns out to be very challenging in general when the repairing node has multiple antennas, i.e., $n_{r} \geq 2$. Almost all existing works such as [38, 47] consider only the case $n_{r}=1$. In [39] Yang and Belfiore investigated the DMT for the MIMO-NAF protocol and provided a lower bound for such DMT. Their result can be modified to yield a lower bound for $d_{2}(r)$. We will comment more on that particular lower bound at the end of this subsection.

To provide bounds on the DMT $d_{2}(r)$ for general values of $n_{r}$, let $U$ be an $\left(n_{r} \times n_{r}\right)$ unitary matrix such that $U \underline{h}_{1}=\left[\begin{array}{llll}\left\|\underline{h}_{1}\right\| & 0 & \cdots & 0\end{array}\right]^{\top}:=\underline{h}$.

For $H_{i}$ defined in (22), $i=2, \ldots, K$, we get

$$
\operatorname{diag}(U, U) H_{i}=\left[\begin{array}{ll}
U & \\
& U
\end{array}\right] H_{i}=\left[\begin{array}{cc}
\underline{h} & \underline{0} \\
a_{i} g_{i} \underline{\ell}_{i} & \underline{h}
\end{array}\right]=S_{i},
$$

where $\underline{\ell}_{i}=U \underline{h}_{i}$ has the same probability density function as $\underline{h}_{i}, i=2, \ldots, K$. Let $\Sigma_{i}:=I_{n_{r}}+\left|a_{i}\right|^{2} \underline{h}_{i} \underline{h}_{i}^{\dagger}$. Clearly, we have the following partial ordering for positive-definite matrices,

$$
I_{n_{r}} \prec \Sigma_{i} \prec\left(1+\left|a_{i}\right|^{2}|| \underline{h}_{i} \|^{2}\right) I_{n_{r}}=\left(1+\left|a_{i}\right|^{2}|| \underline{\ell}_{i} \|^{2}\right) I_{n_{r}},
$$

which in turn implies $\frac{1}{1+\left|a_{i}\right|^{2}|| \underline{\ell}_{i}||^{2}} I_{n_{r}} \prec \Sigma_{i}^{-1} \prec I_{n_{r}}$. With the above, $I(\underline{x} ; \underline{y} \mid H)$ can be upper bounded by

$$
\begin{aligned}
& I \underline{\underline{x}} ; \underline{y} \mid H) \\
& \leq \sum_{i=2}^{K} \log _{2} \operatorname{det}\left(I_{2}+\mathrm{SNR} H_{i}^{\dagger} H_{i}\right) \\
& =\sum_{i=2}^{K} \log _{2}\left[\left(1+\mathrm{SNR} \|\left.\underline{h}\right|^{2}\right)^{2}+\mathrm{SNR}\left|a_{i} g_{i}\right|^{2}|| \underline{\ell}_{i} \|^{2}\right. \\
& \left.+\operatorname{SNR}^{2}\left|a_{i} g_{i}\right|^{2} \|\left.\underline{h}\right|^{2} \sum_{j=2}^{n_{r}}\left|\ell_{i, j}\right|^{2}\right] .
\end{aligned}
$$


Similarly, set $c_{i}=\frac{1}{1+\left.\left|a_{i}\right|^{2}\left|\ell_{i}\right|\right|^{2}}$, and $I(\underline{x} ; \underline{y} \mid H)$ is lower bounded by

$$
\begin{aligned}
& I(\underline{x} ; \underline{y} \mid H) \\
& \geq \sum_{i=2}^{K} \log _{2} \operatorname{det}\left(I_{2}+\operatorname{SNRS}_{i}^{\dagger}\left[\begin{array}{lll}
I_{n_{r}} & \\
& c_{i} I_{n_{r}}
\end{array}\right] S_{i}\right) \\
& =\sum_{i=2}^{K} \log _{2}\left[1+\left(1+c_{i}\right) \mathrm{SNR}\|\underline{h}\|^{2}+c_{i}\left|a_{i} g_{i}\right|^{2}\left\|\underline{\ell}_{i}\right\|^{2} \mathrm{SNR}\right. \\
& \left.+c_{i} \mathrm{SNR}^{2}\|\underline{\underline{h}}\|^{4}+c_{i}^{2}\left|a_{i} g_{i}\right|^{2} \mathrm{SNR}^{2}\|\underline{h}\|^{2} \sum_{j=2}^{n_{r}}\left|\ell_{i, j}\right|^{2}\right] .
\end{aligned}
$$

Equations (27) and (29) then yield the following theorem for bounding the DMT $d_{2}(r)$ for Scheme 2.

Theorem 3. The DMT $d_{2}(r)$ of Scheme 2 for a general number $n_{r} \geq 1$ of receive antennas at the repairing node has the following upper bound $d_{2, U}(r)$ and lower bound $d_{2, L}(r)$ :

$$
\begin{gathered}
d_{2, U}(r):=\inf _{g} \sup _{b \leq g} \inf _{\left(\alpha, \beta_{1}, \beta_{2}\right) \in \mathcal{A}_{U}(r, b, g)} n_{r} \alpha+(K-1) \beta_{1} \\
+\left(n_{r}-1\right)(K-1) \beta_{2}+(K-1) g
\end{gathered}
$$

$$
\begin{array}{r}
d_{2, L}(r):=\inf _{g} \sup _{b \leq g} \inf _{\left.b, \beta_{1}, \beta_{2}\right) \in \mathcal{A}_{L}(r, b, g)} n_{r} \alpha+(K-1) \beta_{1} \\
+\left(n_{r}-1\right)(K-1) \beta_{2}+(K-1) g
\end{array}
$$

where

$$
\begin{aligned}
& \mathcal{A}_{U}(r, b, g) \\
& =\left\{\alpha, \beta_{1}, \beta_{2} \in[0,1]: \max \left\{\begin{array}{l}
2(1-\alpha), \\
1+b-g-\min \left\{\beta_{1}, \beta_{2}\right\} \\
2+b-g-\beta_{2}
\end{array}\right\} \leq 2 K r\right\}
\end{aligned}
$$

and

$$
\begin{aligned}
& \mathcal{A}_{L}(r, b, g) \\
& =\left\{\alpha, \beta_{1}, \beta_{2} \in[0,1]: \max \left\{\begin{array}{l}
1-\alpha, \\
2-2 \alpha-(b-\beta)^{+}, \\
1-\beta+b-g-(b-\beta)^{+}, \\
2-\alpha+b-g-\beta_{2}-2(b-\beta)^{+}
\end{array}\right\} \leq 2 K r\right\} .
\end{aligned}
$$

Proof. Note that the random variables $g_{i}$ 's are i.i.d., hence there is no need to distinguish them in (27) and (29) when deriving the DMT. The same holds also true for $a_{i}, \underline{\ell}_{i}$, and its elements $\ell_{i, j}$ for $i=2, \ldots, K$. Thus, we set $\left|a_{i}\right|^{2} \doteq \mathrm{SNR}^{b},\left|g_{i}\right|^{2} \doteq \mathrm{SNR}^{-g},\|h\|^{2} \doteq \mathrm{SNR}^{-\alpha},\left\|\underline{\ell}_{i}\right\|^{2} \doteq$ $\mathrm{SNR}^{-\beta}$, and $\left|\ell_{i, j}\right|^{2} \doteq \mathrm{SNR}^{-\beta_{j}}$ with $\beta=\min _{j=1, \ldots, n_{r}} \beta_{j}$. Moreover, we note that $\|\underline{h}\|^{2}$ is a $\chi^{2}$ random variable with $2 n_{r}$ degrees of freedom, hence it contributes the term $n_{r} \alpha$ to (27). Each $\underline{\ell}_{i}$ consists of $n_{r}$ i.i.d. $\mathbb{C N}(0,1)$ complex random variables, and there is no need to distinguish $\ell_{i, j}$ for $i=2, \ldots, K$ and for $j=2, \ldots, n_{r}$ as can be seen from (27) and (29). Hence, we can set $\left|\ell_{i, j}\right|^{2} \doteq \mathrm{SNR}^{-\beta_{2}}$ for $i=2, \ldots, K$ and for $j=2, \ldots, n_{r}$. Similarly, there is no need to distinguish $\ell_{i, 1}$ for $i=2, \ldots, K$, hence we set $\left|\ell_{i, 1}\right|^{2} \doteq \mathrm{SNR}^{-\beta_{1}}$ for $i=2, \ldots, K$. Finally, note that $\left|g_{i}\right|^{2}$ and $\left|\ell_{i, j}\right|^{2}$ are i.i.d. $\chi^{2}$ random variables with two degrees of freedom. Plugging the above into (27) and (29) and applying the Laplace principle as in [27] yield the desired upper and lower bounds (30) and (31).

In Fig. 9, we plot the DMT bounds $d_{2, L}(r)$ and $d_{2, U}(r)$ of Scheme 2 as well as the DMT $d^{*}{ }_{1,2,10}(r)$ with $K=10$ helper nodes, $n_{t}=1$ and $n_{r}=2$. While there is a gap between bounds $d_{2, L}(r)$ and $d_{2, U}(r)$ when the multiplexing gain $r$ is small, it can be clearly seen that Scheme 2 can offer a better DMT performance than $d^{*}{ }_{1,2,10}(r)$ when $r$ is small. Regarding the sharpness of $d_{2, L}(r)$ and $d_{2, U}(r)$, let us focus on the case when $r$ is approaching zero from the right, i.e., when $r \downarrow 0$. Note that there are nine SISO channels from helper node 1 to the remaining helper nodes, and the channel between node 1 and the repairing node is a $(1 \times 2)$ SIMO channel. Therefore, the communication to the repairing node would be in outage if the nine SISO channels and the $(1 \times 2)$ SIMO channel are all in deep fade, thereby yielding a maximal diversity order of $9+2=11$. We therefore conclude that the upper bound $d_{2, U}(r)$ can be further improved.

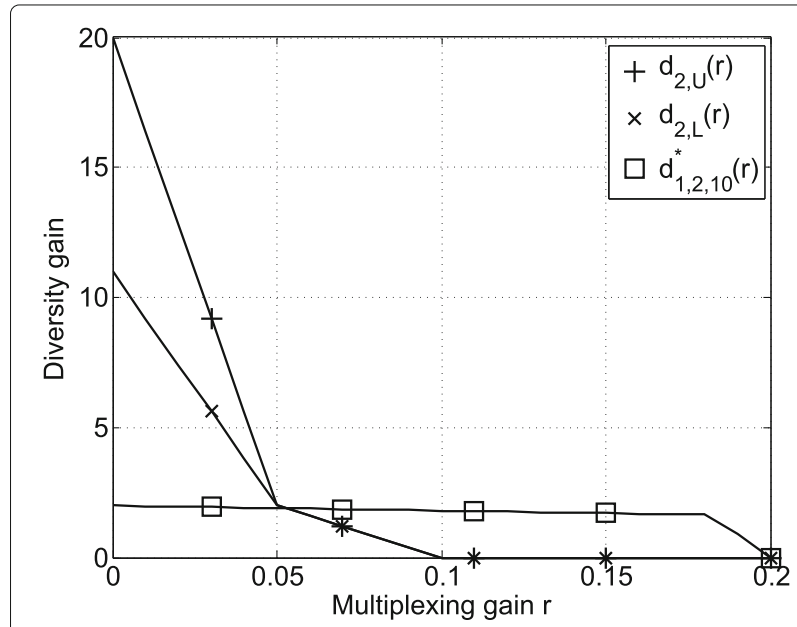

Fig. 9 Scheme 2: DMT bounds and comparison. DMT performances achieved by Scheme 2 (lower bound and the first upper bound) and MIMO-MAC for $K=10$ helper nodes, $n_{t}=1$, and $n_{r}=2$ 
As mentioned earlier, Yang and Belfiore ([39], Theorem 2) provided a lower bound on the DMT for MIMO-NAF protocol. Their bound can be modified to become a lower bound for $d_{2}(r)$ and has the following form

$$
d_{2, \mathrm{~L}, \mathrm{YB}}(r)=n_{r} \cdot(1-K r)^{+}+(K-1) \cdot d_{\mathrm{RP}}(2 K r)
$$

where $d_{\mathrm{RP}}(r)$ is the DMT for the Rayleigh product channel $\underline{h}_{i} \cdot g_{i}$, and an exact expression for $d_{\mathrm{RP}}(r)$ can be found in ([39], Proposition 1).

In Fig. 10, we compare our lower bound $d_{2, L}(r)$ to the lower bound $d_{2, \mathrm{~L}, \mathrm{YB}}(r)$ for the case $n_{t}=1, n_{r}=2$ and $K=10$. It can be clearly seen that, in this case, our bound is shaper than the bound (34).

\subsection{Another upper bound on $d_{\mathbf{2}}(r)$ with general $n_{r}$}

To obtain another upper bound on the instantaneous mutual information $I(\underline{x} ; y \mid H)$, we consider the situation that the repairing node has further knowledge of $r_{i, t-1}=$ $g_{i} x_{t-1}+z_{i}$ when $t=2,4, \ldots, N$ and $i=\frac{t}{2}+1$. In this case, define

$$
\underline{y}_{t}^{\prime}=\underline{h}_{1} x_{t}+w_{t}, \quad t=1,2, \ldots, N
$$

Writing $\underline{y}_{t}=\underline{y}_{t}^{\prime}+a_{i} \underline{h}_{i} r_{i, t-1}$ for $t=2(i-1)$, it follows that

$$
I(\underline{x} ; \underline{y} \mid H) \leq I\left(\underline{x} ; \underline{y}_{1}^{\prime}, \ldots, \underline{y}_{N}^{\prime}, r_{2,1}, r_{3,3}, \ldots, r_{K, N-1} \mid H\right)
$$

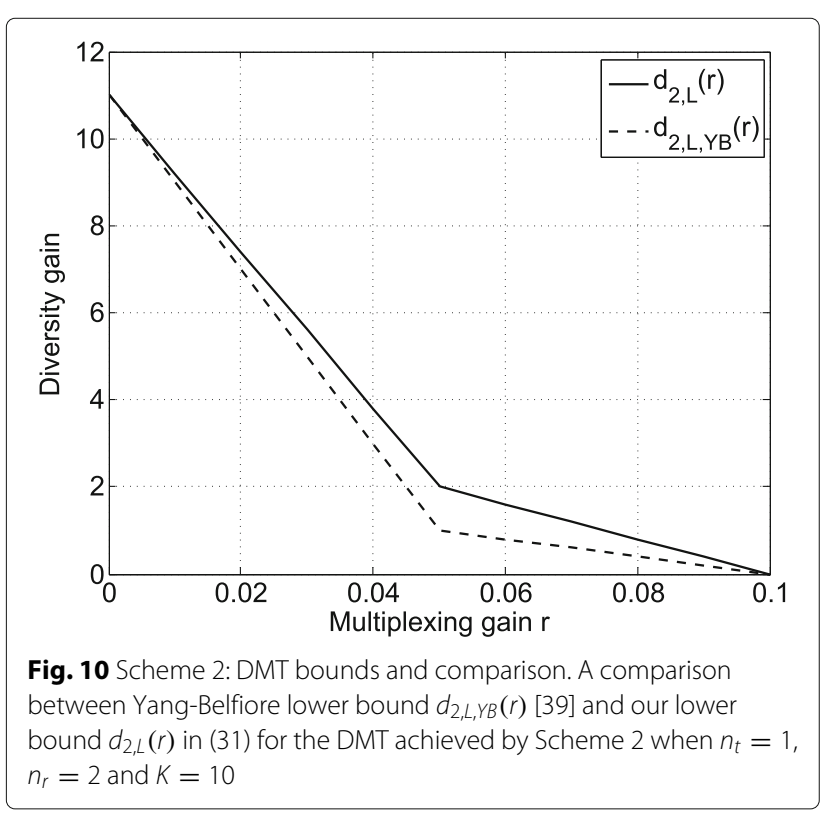

and the upper bound has a much simpler expression than $I(\underline{x} ; \underline{y} \mid H)$. To see this, formulate the received vectors as

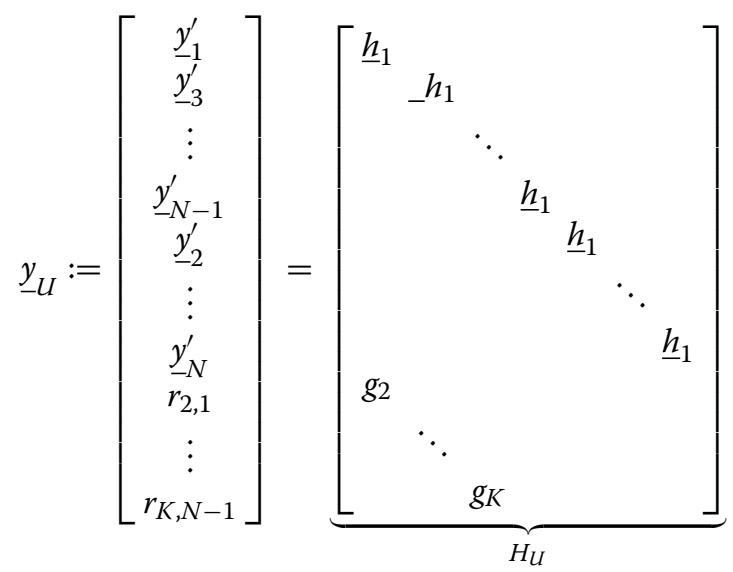$$
\left[\begin{array}{c}
x_{1} \\
x_{3} \\
\vdots \\
x_{N-1} \\
x_{2} \\
x_{4} \\
\vdots \\
x_{N}
\end{array}\right]+\left[\begin{array}{c}
\underline{w}_{1} \\
\underline{w}_{3} \\
\vdots \\
\underline{w}_{N-1} \\
\underline{w}_{2} \\
\vdots \\
\underline{w}_{N} \\
z_{2} \\
\vdots \\
z_{K}
\end{array}\right]
$$

then

$$
H_{U}^{\dagger} H_{U}=\left[\begin{array}{llll}
\left\|\underline{h}_{1}\right\|^{2}+\left|g_{2}\right|^{2} & & & \\
& \ddots & & \\
& & \left\|\underline{h}_{1}\right\|^{2}+\left|g_{K}\right|^{2} & \\
& & \left\|\underline{h}_{1}\right\|^{2} I_{K-1}
\end{array}\right] .
$$

This implies that

$$
\begin{aligned}
& I(\underline{x} ; \underline{y} \mid H) \\
& \leq I\left(\underline{x} ; \underline{y}_{U} \mid H\right) \\
& =(K-1) \log _{2}\left(1+\mathrm{SNR}\left\|\underline{h}_{1}\right\|^{2}\right) \\
& \quad+\sum_{i=2}^{K} \log _{2}\left(1+\operatorname{SNR}\left(\left\|\underline{h}_{1}\right\|^{2}+\left|g_{i}\right|^{2}\right)\right) .
\end{aligned}
$$

Hence, the outage probability for the second scheme is lower bounded by 


$$
\begin{aligned}
& \operatorname{Pr}\left\{H: \sup _{\left|a_{i}\right|^{2} \leq \frac{\mathrm{SNR}}{1+\mathrm{SNR}\left|g_{i}\right|^{2}}} I(\underline{x} ; \underline{y} \mid H)<2 K(K-1) r \log _{2} \mathrm{SNR}\right\} \\
& \geq \operatorname{Pr}\left\{H: I\left(\underline{x} ; \underline{y}_{U} \mid H\right)<2 K(K-1) r \log _{2} \mathrm{SNR}\right\} \\
& \doteq \mathrm{SNR}^{-d_{2, H^{\prime}}(r)} .
\end{aligned}
$$

Theorem 4. The DMT $d_{2}(r)$ for Scheme 2 for a general number $n_{r} \geq 1$ of receive antennas at the repairing node is upper bounded by

$d_{2, U^{\prime}}(r)= \begin{cases}\left(n_{r}+K-1\right)(1-K r)^{+}, & \text {if } n_{r} \geq K-1, \\ 2 n_{r}(1-K r)^{+}+\left(K-1-n_{r}\right)(1-2 K r)^{+}, & \text {if } n_{r} \leq K-1 .\end{cases}$

Proof. Similar to the proof of Theorem 3, it is unnecessary to distinguish the random variables $g_{i}$ in (39) for $i=2, \ldots, K$ when calculating the DMT. Thus, let $\left\|\underline{h}_{1}\right\|^{2}=$ $\mathrm{SNR}^{-\alpha}$ and $\left|g_{i}\right|^{2}=\mathrm{SNR}^{-\beta}$. Note $\left\|\underline{h}_{1}\right\|^{2}$ is a $\chi^{2}$ random variable with $2 n_{r}$ degrees of freedom and $\left|g_{i}\right|^{2}$ is a $\chi^{2}$ random variable with 2 degrees of freedom. Plugging the above into (41) and applying the Laplace principle as in [27] gives

$$
d_{2, U^{\prime}}(r)=\inf _{\mathcal{B}(r)} n_{r} \alpha+(K-1) \beta,
$$

where

$$
\begin{aligned}
\mathcal{B}(r) & =\{\alpha, \beta \in[0,1]: 1-\alpha+\max \{1-\alpha, 1-\beta\} \leq 2 K r\} \\
& =\left\{\alpha, \beta \in[0,1]: 2(1-\alpha)+(\alpha-\beta)^{+} \leq 2 K r\right\} .
\end{aligned}
$$

Solving the above optimization problem gives the desired result.

In Fig. 11, we plot $d_{2, L}(r), d_{2, U}(r)$, and $d_{2, U^{\prime}}(r)$ for the second proposed scheme with $K=10$ helper nodes, $n_{t}=$ 1 and $n_{r}=2$. It can be seen that $d_{2, L}(r)=d_{2, U^{\prime}}(r)$ for all values of $r$, hence we have $d_{2}(r)=d_{2, L}(r)=d_{2, U^{\prime}}(r)$ in this case.

\subsection{Remarks on the complexity exponents of Scheme 2}

Determining the complexity exponents of the second scheme requires much more effort than determining the DMT. At least two major difficulties must be resolved before any identification of complexity exponents is possible. Notice that the notion of complexity exponents resides in an actual construction of space-time codes for the scheme, and that the complexity exponents can vary from one code to another. Codes with a smaller complexity exponent are more favorable in practice, provided that the codes are optimal in the DMT sense, i.e., achieve the DMT $d_{2}(r)$. Therefore, we have to at least identify a space-time code for Scheme 2 first. In [39], Yang and Belfiore provided a systematic construction of space-time

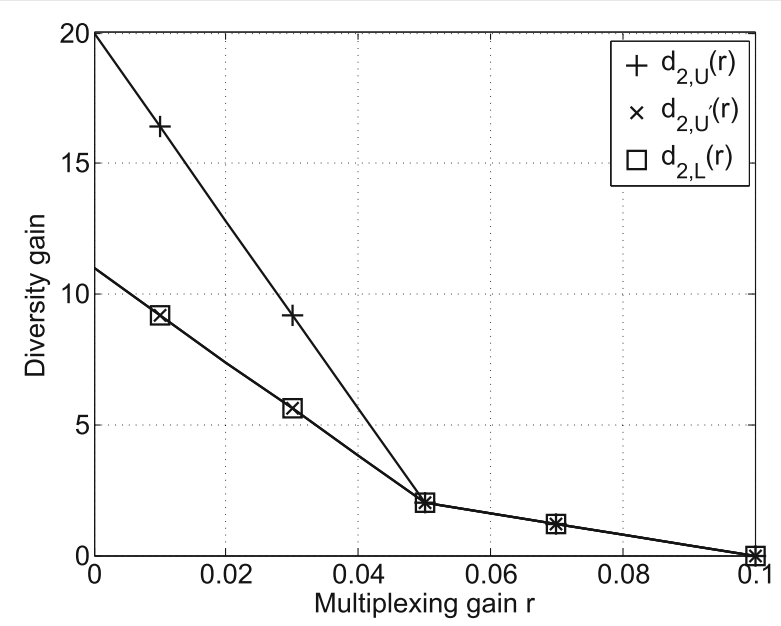

Fig. 11 Scheme 2: improved DMT bounds. DMT performances achieved by Scheme 2 (two upper bounds and a lower bound) for $K=10$ helper nodes, $n_{t}=1$, and $n_{r}=2$

codes that is approximately universal [48] for NAF-based cooperative relay communications. It is certainly possible to adapt their construction to the transmission using Scheme 2.

The second issue complicating the investigation of complexity exponents arises from the need of an exact characterization of eigenvalues of the matrices $H_{i}^{\dagger} K_{i}^{-1} H_{i}$ for $i=2, \ldots, K$, appearing in (21). Determining these eigenvalues is particularly difficult. It is in fact the main reason preventing us from obtaining an exact expression for $d_{2}(r)$ in previous subsections, and we are only able to provide bounds on $d_{2}(r)$ in this paper.

Nevertheless, it can be seen from (20) that the equivalent channel matrix $H$ is of size $\left(N n_{r} \times N\right)$ and has linearly independent columns with probability 1 . This implies that when applying a sphere decoder to decode the codes-for instance, the code constructed by Yang and Belfiore [39] transmitted using Scheme 2, the QR decomposition of the matrix $H$ would result in an upper triangular matrix $R$; hence, there is no ambiguity to be resolved prior to processing the root of the sphere decoding tree. Therefore, the code must be efficiently sphere decodable.

\section{Scheme 3: $n_{t}=1, n_{r} \geq 2$, and $K$ helper nodes}

In the previous section, we presented a powerful scheme that makes a good use of the inter-helper links to improve the DMT performance of DSS repair transmission. The scheme allows one helper node to transmit information in each phase, and the remaining helper nodes are regarded as relays. Furthermore, we have introduced a novel technique that allows us to upper-bound the DMT for the NAF protocol in a cooperative relay network with multiple antennas at the repairing node. In this section, we will present our third scheme, which can be seen as an 
enhancement of Schemes 1 and 2 and can provide a further improvement on the DMT performance.

The third proposed scheme concerns the case $n_{t}=1$, $n_{r} \geq 2$ and $K$ helper nodes. It allows $L$ helper nodes, $L \leq \min \left\{n_{r}, K-1\right\}$, to transmit simultaneously and non-cooperatively to the repairing node as well as to the remaining $(K-L)$ helper nodes, which will function as relays ${ }^{9}$ in the network. To achieve an average multiplexing gain $r$, each of the selected $L$ helper nodes must transmit at a higher multiplexing gain of $\frac{K}{L} r$. In particular, we could later seek to improve the overall DMT performance by optimizing over the choices of $L$. Therefore, $L$ can actually be a function of the multiplexing gain $r$.

Given $L$, the third scheme consists of $\left(\begin{array}{l}K \\ L\end{array}\right)$ phases, one for each possible $L$-subset $\mathcal{L}=\left\{i_{1}, \ldots, i_{L}\right\}$ of $\mathcal{K}$, where $\mathcal{K}=$ $\{1,2, \ldots, K\}$ is the set of helper nodes. The helper nodes in set $\mathcal{L}$ transmit simultaneously and non-cooperatively throughout the phase, which has a duration of $N=$ $2(K-L)$ channel uses. The remaining nodes in $\mathcal{K} \backslash \mathcal{L}=$ $\left\{j_{1}, \ldots, j_{K-L}\right\}$ will function as relays following the NAF protocol. Details of this scheme are given as below, and a pictorial description of this scheme is given in Fig. 12.

\section{Scheme 3}

1: Let $L$ be an integer with $1 \leq L \leq \min \left\{n_{r}, K-1\right\}$.

2: for each $\mathcal{L}=\left\{i_{1}, \ldots, i_{L}\right\} \subseteq \mathcal{K}$ do

3: $\quad$ for $t=1,2, \ldots, N=2(K-L)$ do

4: $\quad$ At the $t$ th channel use of the phase associated with $\mathcal{L}$, each helper node $i_{\ell} \in \mathcal{L}$ broadcasts a signal $x_{i_{\ell}, t}$, with $\mathbb{E}\left|x_{i_{\ell}, t}\right|^{2} \leq \mathrm{SNR}$, to the nodes in $\mathcal{K} \backslash \mathcal{L}$ and to the repairing node

5: $\quad$ Helper-node $j_{s} \in \mathcal{K} \backslash \mathcal{L}, s=1, \ldots,(K-L)$, receives the following signal when $t$ is odd

$$
r_{j_{s}, t}=\sum_{\ell=1}^{L} g_{j_{s}, i_{\ell}} x_{i_{\ell}, t}+z_{j_{s}, t},
$$

where $g_{j_{s}, i_{\ell}}$ and $z_{j_{s}, t}$ are i.i.d. $\mathbb{C N}(0,1)$ random variables defined in (2). When $t=$ $2 s$, node $j_{s}$ broadcasts the signal $a_{j_{s}} r_{j_{s}, 2 s-1}$, where $a_{j_{s}}$ is chosen such that

$$
\left|a_{j_{s}}\right|^{2} \leq \frac{\mathrm{SNR}}{1+\operatorname{SNR} \sum_{\ell=1}^{L}\left|g_{j_{s}, i_{\ell}}\right|^{2}} .
$$

6: $\quad$ The signal received at the repairing node at the $t$-th channel use of the phase associated with $\mathcal{L}$ is

$$
\underline{y}_{t}= \begin{cases}\sum_{\ell=1}^{L} \underline{h}_{i_{\ell}} x_{i, t}+\underline{w}_{t}, & t \text { odd } \\ \sum_{\ell=1}^{L} \underline{\underline{h}}_{i_{\ell}} x_{i_{\ell}, t}+\underline{h}_{j_{s}} a_{j_{s}} r_{j_{s}, t-1}+\underline{w}_{t}, t=2 s\end{cases}
$$

\footnotetext{
7: $\quad$ end for
}

8: end for

\subsection{DMT analysis for Scheme 3}

The communication channel deduced from Scheme 3 resembles the multiple-access relay channel (MARC), which was first introduced by Kramer and van Wijngaarden [49]. The DMTs for the two-user and single-relay MARC - in terms of our notation this means $n_{t}=1$, $n_{r}=1, K=3$ and $L=2$-using various protocols have been studied in the past. For instance, Azarian et al. [50] investigated the DMT for such MARC using the dynamic-decode-and-forward (DDF) strategy, and Yuksel and Erkip [51] focused on the compress-forward (CF) protocol. Furthermore, a protocol similar to Scheme 3 was proposed in [40] and was termed multiple-access amplify-and-forward (MAF), which is a variation of the NAF protocol. It was found in [40] that the MAF outperforms the DDF in the high multiplexing gain regime and the CF protocol [51] in the low multiplexing gain regime when $n_{t}=1, n_{r}=1, K=3$, and $L=2$. The MAF thus provides a nice balance between complexity and performance.

Scheme 3 considers a much more complicated scenario than the one in [40], with $n_{t}=1$, and general values of $n_{r}, K$ and $L \leq \min \left\{n_{r}, K-1\right\}$. To the best of our knowledge, the DMT analysis for the MAF protocol has never been taken to such complexity level. On the other hand, our novel bounding technique employed in the proof of Theorem 4 is extremely powerful and enables us to analyze the DMT for general MARC using the MAF protocol.

To this end, for any subset $\mathcal{U}=\left\{u_{1}, \ldots, u_{k}\right\} \subseteq \mathcal{L}$ of the selected helper nodes, let $\mathcal{E}_{\mathcal{U}}$ denote the event that helper nodes $u_{1}, \ldots, u_{k}$ are in outage. The probability for $\mathcal{E}_{\mathcal{U}}$ is given by

$$
\begin{aligned}
& \operatorname{Pr}\left\{\mathcal{E}_{\mathcal{U}}\right\} \\
= & \operatorname{Pr}\left\{\left\{\underline{h}_{i}\right\}_{i=1}^{K},\left\{g_{i, j}\right\}_{i, j=1}^{K}: \sup _{\substack{a_{j, s} \\
s=1, \ldots, K-L}} I\left(\left\{x_{u, t}: u \in \mathcal{U}\right\}_{t=1}^{N} ; \underline{y}_{1}, \ldots, \underline{y}_{N} \mid\right.\right. \\
& \left.\left.\left\{x_{u, t}: u \in \mathcal{L} \backslash \mathcal{U}\right\}_{t=1}^{N},\left\{\underline{h}_{i}\right\}_{i=1}^{K},\left\{g_{i, j}\right\}_{i, j=1}^{K}\right)<\frac{N K}{L} r k \log _{2} \operatorname{SNR}\right\},
\end{aligned}
$$

where $N=2(K-L)$. The overall outage probability for the third proposed scheme with given $L$ is

$$
P_{\text {out }, 3}(L, r):=\operatorname{Pr}\left\{\bigcup_{\mathcal{U} \subseteq \mathcal{L}} \mathcal{E}_{\mathcal{U}}\right\} \doteq \max _{\mathcal{U} \subseteq \mathcal{L}} \operatorname{Pr}\left\{\mathcal{E}_{\mathcal{U}}\right\} \doteq \mathrm{SNR}^{-d_{3}(L, r)}
$$

The technique introduced in Section 5.4 can be applied to yield the following upper bound on $d_{3}(L, r)$. 


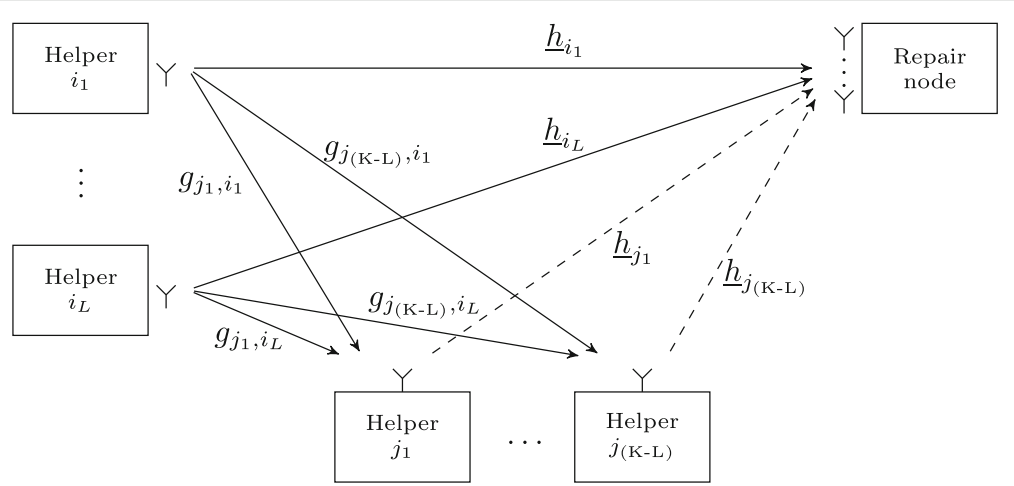

Fig. 12 Channel model of Scheme 3. Channel model for Scheme 3 at the phase associated with $\mathcal{L}=\left\{i_{1}, \ldots, i_{L}\right\}$

Theorem 5. The DMT $d_{3}(L, r)$ can be upper bounded as

$$
\begin{aligned}
d_{3}(L, r) \leq d_{3, U}(L, r):= & \min _{k=1, \ldots, L} \inf _{\mathcal{A}(L, k, r)} \sum_{i=1}^{k}\left[2 i-1+\left(n_{r}-k\right)\right] \alpha_{i} \\
& +(K-L)\left[\sum_{j=1}^{k} \beta_{j}+\left(n_{r}-k\right) \beta_{k+1}\right],
\end{aligned}
$$

where

$$
\begin{aligned}
& \mathcal{A}(L, k, r) \\
& :=\left\{\begin{array}{l}
\alpha_{1}, \cdots \alpha_{k}, \beta_{1}, \cdots \beta_{k+1}: \\
1 \geq \alpha_{1} \geq \alpha_{2} \geq \cdots \geq \alpha_{k} \geq 0, \\
\alpha_{i} \geq \beta_{i} \geq 0, \quad i=1,2, \ldots, k, \\
\beta_{k+1} \geq 0 \text { and } \beta_{k+1}=0 \text { if } n_{r}=k, \\
\sum_{i=1}^{k}\left(1-\alpha_{i}\right)+\frac{1}{2} \max \left\{\alpha_{1}-\beta_{1}, \ldots, \alpha_{k}-\beta_{k}, \beta_{k+1}\right\}<\frac{K r k}{L}
\end{array}\right\} .
\end{aligned}
$$

Proof. Given any $\mathcal{U}=\left\{u_{1}, \ldots, u_{k}\right\} \subseteq \mathcal{L}$ of selected helper nodes, we first reformulate the channel inputoutput relations (45) in matrix form. For the sake of notational convenience, we set $r_{s}=r_{j_{s}, 2 s-1}, z_{s}=z_{j_{s}, 2 s-1}$,

$\underline{x}_{t}:=\left[\begin{array}{c}x_{u_{1}, t} \\ \vdots \\ x_{u_{k}, t}\end{array}\right], H_{\mathcal{U}}=\left[\begin{array}{lll}\underline{h}_{u_{1}} & \cdots & \underline{h}_{u_{k}}\end{array}\right]$, and $\underline{g}_{s}=\left[\begin{array}{c}g_{j_{s}, u_{1}} \\ \vdots \\ g_{j_{s}, u_{k}}\end{array}\right]$,

for $j_{s} \in \mathcal{K} \backslash \mathcal{L}$ and $s=1, \ldots,(K-L)$. Following the same approach as in Section 5.4, we assume the repairing node has further knowledge of $r_{s}$ for $s=1, \ldots,(K-L)$; therefore, it knows $\underline{y}_{t}^{\prime}=H_{\mathcal{U}} \underline{x}_{t}+\underline{w}_{t}$ for $t=1,2, \ldots, N$. We then have

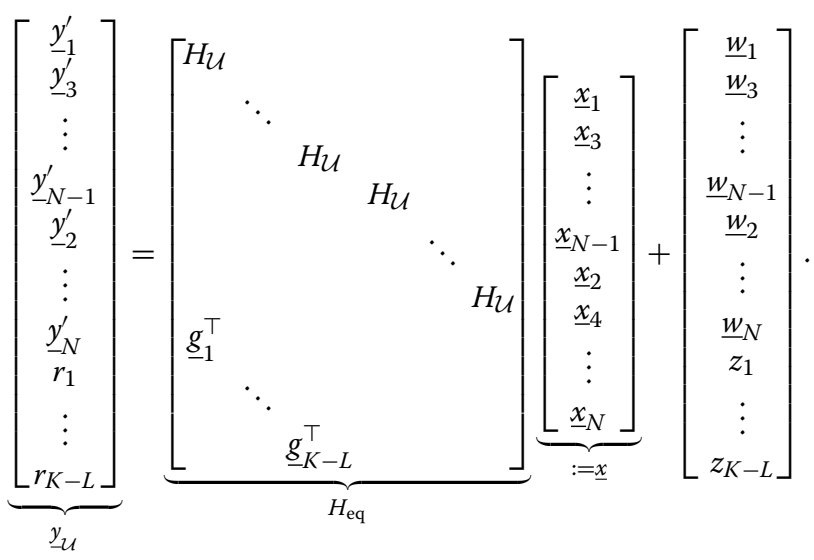

It follows that

$$
\begin{aligned}
& I\left(\left\{x_{u, t}: u \in \mathcal{U}\right\}_{t=1}^{N} ; \underline{y}_{1}, \ldots, \underline{y}_{N} \mid\left\{x_{u, t}: u \in \mathcal{K} \backslash \mathcal{U}\right\}_{t=1}^{N},\right. \\
& \left.\quad\left\{\underline{h}_{i}\right\}_{i=1}^{K},\left\{g_{i, j}\right\}_{i, j=1}^{K}\right) \\
& \leq I\left(\underline{x} ; \underline{y}_{\mathcal{U}} \mid H_{\mathrm{eq}}\right) \\
& =\frac{N}{2} \log _{2} \operatorname{det}\left(I_{k}+\mathrm{SNR} H_{\mathcal{U}}^{\dagger} H_{\mathcal{U}}\right) \\
& \quad+\sum_{s=1}^{\frac{N}{2}} \log _{2} \operatorname{det}\left(I_{k}+\mathrm{SNR} H_{\mathcal{U}}^{\dagger} H_{\mathcal{U}}+\mathrm{SNR} \underline{g}_{s}^{*} \underline{g}_{s}^{\top}\right) .
\end{aligned}
$$

Let $H_{\mathcal{U}}^{\dagger} H_{\mathcal{U}}=E \Lambda E^{\dagger}$ be the eigen-decomposition of $H_{\mathcal{U}}^{\dagger} H_{\mathcal{U}}$, where $E$ is a $(k \times k)$ unitary matrix, $\Lambda=$ $\operatorname{diag}\left(\lambda_{1}, \cdots, \lambda_{k}\right)$, and $0<\lambda_{1} \leq \cdots \leq \lambda_{k}$ are the nonzero 
ordered eigenvalues of $H_{\mathcal{U}}^{\dagger} H_{\mathcal{U}}$, since $\operatorname{rank}\left(H_{\mathcal{U}}^{\dagger} H_{\mathcal{U}}\right)=k$ with probability one. The instantaneous mutual information $I\left(\underline{x} ; \underline{y}_{\mathcal{U}} \mid H_{\text {eq }}\right)$ can be further simplified to

$$
\begin{aligned}
I & \left(\underline{x} ; \underline{y}_{\mathcal{U}} \mid H_{\mathrm{eq}}\right) \\
= & N \sum_{s=1}^{k} \log _{2}\left(1+\mathrm{SNR} \lambda_{s}\right) \\
& +\sum_{s=1}^{\frac{N}{2}} \log _{2}\left(I_{k}+\left(I_{k}+\mathrm{SNR} \Lambda\right)^{-1} \mathrm{SNR}^{\dagger} \underline{g}_{s}^{*} \underline{g}_{s}^{\top} E\right) \\
= & N \sum_{s=1}^{k} \log _{2}\left(1+\mathrm{SNR} \lambda_{s}\right) \\
& +\sum_{s=1}^{\frac{N}{2}} \log _{2}\left(1+\sum_{j=1}^{k} \frac{\operatorname{SNR}}{1+\operatorname{SNR} \lambda_{j}}\left|v_{s, j}\right|^{2}+\sum_{j=k+1}^{n_{r}} \operatorname{SNR}\left|v_{s, j}\right|^{2}\right),
\end{aligned}
$$

where we have set $\underline{v}_{s}=E^{\dagger} \underline{g}_{s}^{*}$, which is a length- $n_{r}$ random vector with i.i.d. $\mathbb{C N}(0,1)$ entries. It follows that

$\operatorname{Pr}\left\{\mathcal{E}_{\mathcal{U}}\right\} \geq \operatorname{Pr}\left\{I\left(\underline{x} ; \underline{y}_{\mathcal{U}} \mid H_{\text {eq }}\right)<\frac{N K}{L} r k \log _{2} \mathrm{SNR}\right\} \doteq \mathrm{SNR}^{-d_{\mathcal{U}}(r)}$.

We set

- $\lambda_{s}=\mathrm{SNR}^{-\alpha_{s}}$ for $s=1, \ldots, \frac{N}{2}$, with each $\alpha_{s}$ contributing the term $\left(2 s-1+\left(n_{r}-k\right)\right) \alpha_{s}$ to the overall diversity order.

- $\left|v_{s, j}\right|^{2}=\mathrm{SNR}^{-\beta_{s, j}}=\mathrm{SNR}^{-\beta_{j}}$ for $s=1, \ldots, \frac{N}{2}$ and $j=1, \ldots, k$, since there is no need to distinguish $v_{s, j}$ in these cases when applying the Laplace principle to (53). Each $\beta_{j}, j=1, \ldots, k$, contributes the term $\frac{N}{2} \beta_{j}$ to the overall diversity order.

- $\left|v_{s, j}\right|^{2}=\mathrm{SNR}^{-\beta_{s, j}}=\mathrm{SNR}^{-\beta_{k+1}}$ for $s=1, \ldots, \frac{N}{2}$ and $j=k+1, \ldots, n_{r}$, for the same reason. The factor $\beta_{k+1}$ contributes the term $\frac{N}{2}\left(n_{r}-k\right) \beta_{k+1}$ to the overall diversity order.

It follows from the above that

$$
\begin{aligned}
d_{\mathcal{U}}(r):= & \inf _{\mathcal{A}^{\prime}(L, k, r)} \sum_{i=1}^{k}\left[2 i-1+\left(n_{r}-k\right)\right] \alpha_{i}+(K-L) \sum_{j=1}^{k} \beta_{j} \\
& +(K-L)\left(n_{r}-k\right) \beta_{k+1},
\end{aligned}
$$

where

$$
\begin{aligned}
& \mathcal{A}^{\prime}(L, k, r):= \\
& \left\{\begin{array}{c}
\alpha_{1}, \ldots, \alpha_{k}, \beta_{1}, \ldots, \beta_{k+1} \in \mathbb{R}: \\
\alpha_{1} \geq \alpha_{2} \geq \cdots \geq \alpha_{k} \geq 0, \beta_{1}, \beta_{2}, \ldots, \beta_{k+1} \geq 0, \\
\beta_{k+1}=0 \text { if } n_{r}=k \\
\sum_{i=1}^{k}\left(1-\alpha_{i}\right)^{+}+\frac{1}{2} \max \left\{\left(1-\beta_{j}-\left(1-\alpha_{j}\right)^{+}\right)^{+}, \ldots,\right. \\
\left.\left(1-\beta_{k}-\left(1-\alpha_{k}\right)^{+}\right)^{+}, \beta_{k+1}\right\}<\frac{K r k}{L}
\end{array}\right\}
\end{aligned}
$$

Finally, the upper bound $d_{3, U}(L, r)$ is obtained after minimizing $d_{\mathcal{U}}(r)$ for all possible subsets $\mathcal{U} \subseteq \mathcal{L}$ (or equivalently for all $k=1, \ldots, L)$ and after simplifying the constraints in (55).

By optimizing over all possible $L=1,2, \ldots, \min \left\{n_{r}, K-\right.$ 1) for $d_{3, U}(r)$, we obtain an upper bound on the DMT performance for the third scheme.

Corollary 6. The DMT performance for Scheme 3 is upper bounded by

$$
d_{3, U}(r):=\max _{L=1, \ldots, \min \left\{K-1, n_{r}\right\}} d_{3, U}(L, r)
$$

In Fig. 13, we illustrate the overall picture for the case of $n_{t}=1, n_{r}=2$, and $K=10$. While we do not yet have a lower bound or a tight DMT result, we believe that Scheme 3 is indeed likely to be superior ${ }^{10}$ to all other schemes presented in this paper, namely the TDMA scheme, Schemes 1 and 2.

In particular, we note that the DMT upper bound for Scheme 3 achieves the maximal possible multiplexing gain of $\frac{2}{10}=0.2$, which is the same as the TDMA scheme and the MIMO-MAC. Such possibility for the optimality of Scheme 3 turns out to be generally true, at least from the viewpoint of the upper bound (51). To see this, note that by (51), we have

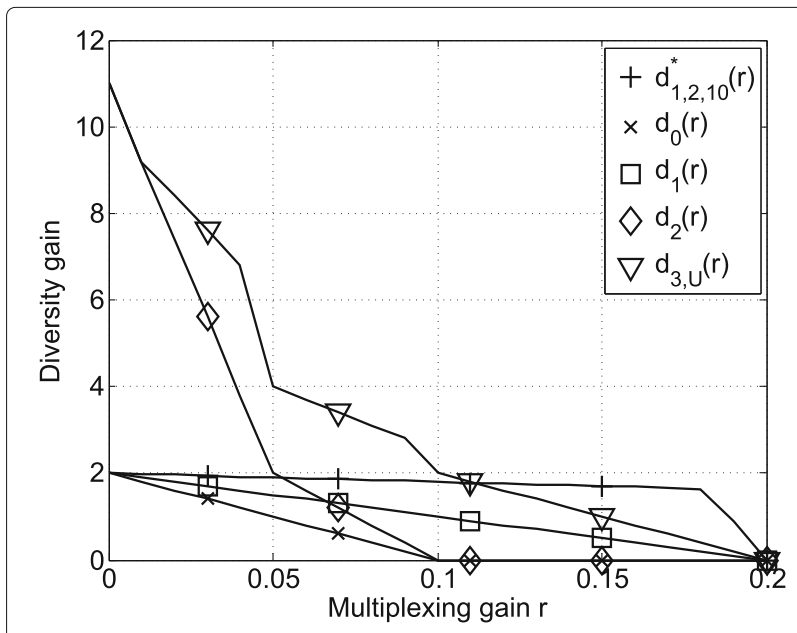

Fig. 13 Overall DMT comparison. DMT performances achieved by the MIMO-MAC, time-sharing scheme and Schemes 1,2, and 3, for $K=10$ helper nodes, $n_{t}=1$, and $n_{r}=2$ 


$$
\begin{aligned}
& \mathbb{E}\left[\frac{I\left(\underline{x} ; \underline{-}_{\mathcal{L}} \mid H_{\mathrm{eq}}\right)}{N \log _{2} \mathrm{SNR}}\right] \\
& =\frac{\mathbb{E} \frac{1}{2} \log _{2} \operatorname{det}\left(I_{L}+\mathrm{SNR} H_{\mathcal{L}}^{\dagger} H_{\mathcal{L}}\right)+\frac{1}{2} \mathbb{E} \log _{2} \operatorname{det}\left(I_{L}+\mathrm{SNR} H_{\mathcal{L}}^{\dagger} H_{\mathcal{L}}+\mathrm{SNR} \underline{g}_{1}^{*} \underline{g}_{1}^{\top}\right)}{\log _{2} \mathrm{SNR}} \\
& =\frac{1}{2} \min \left\{L, n_{r}\right\}+\frac{1}{2} \min \left\{L, n_{r}+1\right\}+o(1),
\end{aligned}
$$

as SNR $\rightarrow \infty$, where the last equality follows from the asymptotic analysis of the degrees-of-freedom (DoF) for the MIMO channel $[27,52]$ and from the fact that $H_{\mathcal{L}}$ is a channel matrix of size $\left(n_{r} \times L\right)$, and $H_{\mathcal{L}}^{\dagger} H_{\mathcal{L}}+g_{1}^{*} g_{1}^{\top}=F F^{\dagger}$ with $F=\left[H_{\mathcal{L}}^{\dagger} \underline{g}_{1}^{*}\right]$ is a matrix of size $\left(L \times\left(n_{r}+1\right)\right)$. Eq. 57 shows that the channel capacity resulting from Scheme 3 equals $L \cdot \log _{2} \mathrm{SNR}+\mathrm{O}\left(\log _{2} \mathrm{SNR}\right)$ in high SNR regime for $L \leq \min \left\{n_{r}, K-1\right\}$, and such an amount of capacity is shared by the $L$ selected helper nodes. In other words, each selected helper node gets $1 \cdot \log _{2} \mathrm{SNR}+\mathrm{O}\left(\log _{2} \mathrm{SNR}\right)$ bits per channel use as the maximal achievable transmission rate. Note that in Scheme 3 the selected helper node must transmit at a higher multiplexing gain $\frac{K}{L} r$ such that the average multiplexing gain equals $r$. This then implies

$$
\frac{K}{L} r \log _{2} \mathrm{SNR} \leq 1 \cdot \log _{2} \mathrm{SNR}+o\left(\log _{2} \mathrm{SNR}\right)
$$

i.e., $r \leq \frac{L}{K}$. Now, with $L=n_{r}<K$ we see that Scheme 3 achieves the maximal possible multiplexing gain of $\frac{n_{r}}{K}$ for each helper node, same as MIMO-MAC [28], where the maximal possible multiplexing gain is given by $\frac{\min \left\{K n_{t}, n_{r}\right\}}{K}=\frac{n_{r}}{K}$.

\section{Conclusions}

The communications within a wireless storage network can be modeled as a multiple-access channel with additional inter-source communication links. Motivated by this observation, we have proposed three physical layer transmission schemes based on different time-sharing and relaying strategies that are suitable for the given channel model. In contrast to the state-of-the-art MAC DMT optimal algebraic space-time codes, our schemes are efficiently sphere-decodable with only one or two antennas. Their DMT performance reaches between the timesharing DMT and the optimal MAC DMT-the one for conventional MIMO-MAC having no inter-source linksin the high-multiplexing gain regime. When the desired multiplexing gain is low, the schemes even outperform the optimal MAC DMT. Naturally, the schemes are also applicable to DSS file reconstruction, as well as to any MAC communications with inter-source links.
In the future, even small devices with very limited power may be equipped with several antennas thanks to massive MIMO at $60 \mathrm{~Hz}$. However, implementation of a practical massive MIMO system still calls for a considerable amount of research efforts regarding pilot design, channel estimation, and code design. Before all that is realized, we believe that the proposed schemes provide a good and efficient alternative.

\section{Endnotes}

Device-to-device (D2D) communication networks provide one such example, see e.g. [53, 54].

Preliminary results related to this work were reported in the Global Wireless Summit 2014 GWS'14 [18] (invited abstract which is considered a preprint), 21st International Symposium on Mathematical Theory of Networks and Systems MTNS'14 [19] (short invited abstract, Scheme 1), and 2014 International Symposium on Information Theory and Its Applications (ISITA) [20] (Schemes 1-3, now combined to Scheme 2). We point out that the numbering of the schemes has been changed so that the schemes previously called 2 and 3 [20] have been combined to Scheme 2, and the new scheme is hence now called Scheme 3 and has not appeared anywhere before. This paper extends the results by additional proofs for the bounds related to Scheme 2, and with a completely new scheme, Scheme 3, that improves upon the other schemes.

BBy efficiently sphere-decodable space-time code we mean that the code can be sphere-decoded without the need of performing an exhaustive search for part of the symbols before starting processing the root of a spheredecoding tree. See discussions in Section 2.2.

BBy half-duplex we mean each node can choose to either transmit or receive, but not both at the same time.

5 Such a comparison might not seem fair to some readers as (5) assumes no inter-helper links. However, the DMT (5) is the best DMT result that can be found in the related literature. 
${ }^{6}$ This latter condition might seem unrealistic in certain (logical) distributed storage codes. However, it would be extremely difficult to determine the mutual information between the helpers and the repairing node if one takes into account the shared information among helper nodes.

${ }^{7} \mathrm{~A}$ lattice is a discrete abelian subgroup of a real or complex vector space, and its rank is given by its rank as a module over $\mathbb{Z}$. By an algebraic lattice we refer to one constructed from a number field extension or a division algebra, see e.g. [29].

It is unfortunate that measuring the exact complexity exponents for these schemes is extremely complicated, and we are unable to complete the task in this paper. Nevertheless, it can still be seen that these schemes can yield efficiently sphere-decodable space-time codes without the need of resolving ambiguities when processing the sphere-decoding tree.

${ }^{9}$ Here we have implicitly assumed $K-L \geq 1$ such that at least one helper node will function as a relay.

${ }^{10} \mathrm{Cf}$. the corresponding upper bound $d_{2, U^{\prime}}(r)=d_{2}(r)$ for Scheme 3 that turned out to be tight.

\section{Competing interests}

The authors declare that they have no competing interests.

\section{Acknowledgements}

C. Hollanti is supported by the Academy of Finland grants \#276031, \#282938, and \#283262, and by Magnus Ehrnrooth Foundation, Finland. D. Karpuk is supported by the Academy of Finland grant \#268364. The support from the European Science Foundation under the ESF COST Action IC1104 is also gratefully acknowledged. The research of H. F. Lu was funded in part by Taiwan Ministry of Science and Technology under Grants MOST 101-2923-E-009-001-MY3 and MOST 103-2221-E-009-043-MY3.

\section{Author details}

${ }^{1}$ Department of Electrical and Computer Engineering, National Chiao Tung University, ED726, 1001 University Rd., 300 Hsinchu, Taiwan. ${ }^{2}$ Department of Mathematics and Systems Analysis, Aalto University, P.O. Box 11100, FI-00076 AALTO (Espoo), Finland.

Received: 3 July 2015 Accepted: 9 March 2016

Published online: 05 May 2016

\section{References}

1. Extracting value from chaos (2011). www.emc.com/collateral/analystreports/idc-extracting-value-from-chaos-ar.pdf, digital Universe Study by the EMC corporation, Accessed 18.11.2015

2. The digital universe of opportunities (2014). http://www.emc.com/ collateral/analyst-reports/idc-digital-universe-2014.pdf, digital Universe Study by the EMC corporation

3. Cisco visual networking index: Global mobile data traffic forecast update, 2014-2019, white paper (2015). http://www.cisco.com/c/en/us/ solutions/collateral/service-provider/ip-ngn-ip-next-generationnetwork/white_paper_c11-481360.pdf

4. F Oggier, A Datta, Coding techniques for repairability in networked distributed storage systems. Found. Trends Commun. Inf. Theory. 9(4), 383-466 (2013)
5. A Lakshman, P Malik, Cassandra: a decentralized structured storage system. SIGOPS Oper. Syst. Rev. 44(2), 35-40 (2010)

6. R Jennings, Cloud computing with the Windows Azure platform. Wiley Publishing (2009)

7. FJ MacWilliams, NJA Sloane, The theory of error-correcting codes. North-Holland (1983)

8. AG Dimakis, B Godfrey, Y Wu, MJ Wainwright, K Ramchandran, Network coding for distributed storage systems. IEEE Trans. Inf. Theory. 56(9), 4539-4551 (2010)

9. KV Rashmi, NB Shah, PV Kumar, Optimal exact-regenerating codes for distributed storage at the MSR and MBR points via a product-matrix construction. IEEE Trans. Inf. Theory. 57(8), 5227-5239 (2011)

10. T Ernvall, SE Rouayheb, C Hollanti, HV Poor, Capacity and security of heterogeneous distributed storage systems. IEEE J. Sel. Areas Commun. 31(12), 2701-2709 (2013)

11. SYE Rouayheb, K Ramchandran, in Proc. 48th Annual Allerton Conference, Sept. 29 2010-Oct. 1 2010. Fractional Repetition Codes for Repair in Distributed Storage Systems, (Monticello, IL, USA, 2010), pp. 1510-1517

12. J Rabaey, in Keynote Address. A brand new wireless day (ASPDAC, Seoul, 2008)

13. J Rabaey, Connectivity brokerage_enabling seamless cooperation in wireless networks. white paper (2010). https://faculty.ozyegin.edu.tr/ aliercan/files/2012/10/Pub6.pdf

14. J Rabaey, in Proc. Symp. VLSI Circuits, June 15-17, 2011. The swarm at the edge of the cloud - a new perspective on wireless, (Honolulu, HI, USA, 2011), pp. 2158-5601

15. E Bastug, M Bennis, M Debbah, Living on the edge: The role of proactive caching in $5 \mathrm{~g}$ wireless networks. IEEE Commun. Mag. 52(8), 82-89 (2014)

16. C Gong, X Wang, On partial downloading for wireless distributed storage networks. IEEE Trans. Signal Process. 60(6), 3278-3288 (2012)

17. KV Rashmi, NB Shah, K Ramchandran, PV Kumar, in Proceedings of the 2012 IEEE International Symposium on Information Theory, ISIT 2012, July 16, 2012. Regenerating codes for errors and erasures in distributed storage, (Cambridge, MA, USA, 2012), pp. 1202-1206

18. C Hollanti, DA Karpuk, A Barreal, HF Lu, Proc. 4th International Conference on Wireless Communications, Vehicular Technology, Information Theory and Aerospace \& Electronic Systems (VITAE), May 11-14, 2014, Aalborg, Denmark. Space-time storage codes for wireless distributed storage systems, pp. 1-5 (2014)

19. A Barreal, C Hollanti, DA Karpuk, H Lu, in Pre-proceedings of the 21st International Symposium on Mathematical Theory of Networks and Systems, MTNS 2014, July 7-11, 2014. Algebraic Codes and a New Physical Layer Transmission Protocol for Wireless Distributed Storage Systems, (Groningen, The Netherlands, 2014)

20. C Hollanti, H Lu, DA Karpuk, A Barreal, in Proc. 2014 IEEE International Sympos ium on Information Theory and its Applications, ISITA 2014, October 26-29, 2014. New relay-based transmission protocols for wireless distributed storage systems, (Melbourne, VIC, Australia, 2014), pp. 585-589

21. TM Cover, JA Thomas, Elements of Information Theory, 2nd. edn. (John Wiley \& Sons, New Jersey, 2006)

22. VTarokh, N Seshadri, AR Calderbank, Space-time codes for high data rate wireless communication: performance criterion and code construction. IEEE Trans. Inf. Theory. 44, 744-765 (1998)

23. P Elia, KR Kumar, SA Pawar, PV Kumar, HF Lu, Explicit space-time codes achieving the diversity-multiplexing gain tradeoff. IEEE Trans. Inf. Theory. 52(9), 3869-3884 (2006)

24. C Hollanti, J Lahtonen, HF Lu, Maximal orders in the design of dense space-time lattice codes. IEEE Trans. Inf. Theory. 54(10), 4493-4510 (2008)

25. JC Belfiore, G Rekaya, E Viterbo, The Golden code: a $2 \times 2$ full-rate space-time code with non-vanishing determinants. IEEE Trans. Inf. Theory. 51(4), 1432-1436 (2005)

26. HF Lu, C Hollanti, R Vehkalahti, J Lahtonen, DMT optimal codes constructions for multiple-access MIMO channel. IEEE Trans. Inf. Theory. 57(6), 3594-3617 (2011)

27. L Zheng, DNC Tse, Diversity and multiplexing: a fundamental tradeoff in multiple antenna channels. IEEE Trans. Inf. Theory. 49(5), 1073-1096 (2003)

28. DNC Tse, P Viswanath, L Zheng, Diversity-multiplexing tradeoff in multiple-access channels. IEEE Trans. Inf. Theory. 50(9), 1859-1874 (2004)

29. F Oggier, JC Belfiore, E Viterbo, Cyclic Division Algebras: a Tool for Space-Time Coding. (Publishers Inc. PO Box 1024 Hanover, MA 02339 USA, 2007) 
30. B Hassibi, $\mathrm{M} \mathrm{Hochwald,} \mathrm{High-rate} \mathrm{codes} \mathrm{that} \mathrm{are} \mathrm{linear} \mathrm{in} \mathrm{space} \mathrm{and} \mathrm{time.}$ IEEE Trans. Inf. Theory. 48(7), 1804-1824 (2002)

31. B Hassibi, H Vikalo, On the sphere-decoding algorithm I, expected complexity. IEEE Trans. Signal Process. 53(8), 2806-2818 (2005)

32. T Tang, H Tien, HF Lu, Selection and rate-adaptation schemes for MIMO multiple-access channels with low-rate channel feedback. IEEE Trans. Inf. Theory. 61(11), 5948-5975 (2015)

33. TW Tang, MK Chen, HF Lu, Improving the DMT performance for MIMO communication with linear receivers. IEEE Trans. Veh. Technol. 62(3), 1189-1200 (2013)

34. D Tse, P Viswanath, Fundamentals of Wireless Communication. (Cambridge, University Press, Cambridge, UK, 2005)

35. J Jaldén, P Elia, Sphere decoding complexity exponent for decoding full-rate codes over the quasi-static MIMO channel. IEEE Trans. Inf. Theory. 58(9), 5785-5803 (2012)

36. HF Lu, P Elia, A Singh, Performance-complexity analysis for MAC ML-based decoding with user selection. IEEE Trans. Signal Process. (2014). arxiv. 1505.07725

37. JN Laneman, GW Wornell, Distributed space-time-coded protocols for exploiting cooperative diversity in wireless networks. IEEE Trans. Inf. Theory. 49(10), 2415-2425 (2003)

38. K Azarian, H El Gamal, P Schniter, On the achievable diversity-multiplexing tradeoff in half-duplex cooperative channels. IEEE Trans. Inf. Theory. 51(12), 4152-4172 (2005)

39. S Yang, JC Belfiore, Optimal space-time codes for the MIMO amplify-and-forward cooperative channel. IEEE Trans. Inf. Theory. 53(2), 647-663 (2007)

40. D Chen, K Azarian, JN Laneman, A case for amplify-forward relaying in the block-fading multiple-access channel. IEEE Trans. Inf. Theory. 54(8), 3728-3733 (2008)

41. MO Damen, HE Gamal, G Caire, On maximum-likelihood detection and the search for the closest lattice point. IEEE Trans. Inf. Theory. $\mathbf{4 9 ( 1 0 ) ,}$ 2389-2402 (2003)

42. MO Damen, H El Gamal, G Caire, in Proc. 2004 IEEE Int. Symp. Inf. Theory. MMSE-GDFE Lattice Decoding for Solving Under-determined Linear Systems With Integer Unknowns, (Chicago, IL, 2004), p. 538

43. LG Barbero, T Ratnarajah, C Cowan, in Proceedings of the IEEE International Conference on Acoustics, Speech, and Signal Processing, ICASSP 2008, March 30 - April 4, 2008, Caesars Palace, Las Vegas, Nevada, USA. A low-complexity soft-mimo detector based on the fixed-complexity sphere decoder, (2008), pp. 2669-2672

44. K Su, Detection and decoding of signals transmitted over linear MIMO channels. PhD thesis, University of Cambridge (2005)

45. J Jaldén, P Elia, DMT optimality of LR-aided linear decoders for a general class of channels, lattice designs, and system models. IEEE Trans. Inf. Theory. 56(10), 4765-4780 (2010)

46. AK Singh, J Jaldén, P Elia, Achieving a vanishing SNR gap to exact lattice decoding at a subexponential complexity. IEEE Trans. Inf. Theory. 58(6), 3692-3707 (2012)

47. P Elia, KVinodh, M Anand, PV Kumar, D-MG tradeoff and optimal codes for a class of AF and DF cooperative communication protocols. IEEE Trans. Inf. Theory. 55( 7), 3161-3185 (2009)

48. STavildar, P Viswanath, Approximately universal codes over slow fading channels. IEEE Trans. Inf. Theory. 52(7), 3233-3258 (2006)

49. G Kramer, AJ van Wijngaarden, in Proc. 2000 IEEE Int. Symp. Inf. Theory. On the White Gaussian Multiple-acess Relay Channel, (Sorrento, Italy, 2000)

50. K Azarian, HE Gamal, P Schniter, On the optimality of the ARQ-DDF protocol. IEEE Trans. Inf. Theory. 54(4), 1718-1724 (2008)

51. M Yuksel, E Erkip, Multiple-antenna cooperative wireless systems: A diversity-multiplexing tradeoff perspective. IEEE Trans. Inf. Theory. 53(10), 3371-3393 (2007)

52. E Telatar, Capacity of multi-antenna Gaussian channels. Europ. Trans. Telecomm. 10(6), 585-595 (1999)

53. J Pääkkönen, C Hollanti, O Tirkkonen, Device-to-device data storage for mobile cellular systems. Device-to-Device (D2D) Communication With and Without Infrastructure, Globecom 2013, arXiv:1309.6123 (2013)

54. J Pääkkönen, C Hollanti, O Tirkkonen, Device-to-device data storage with regenerating codes. 8th International Workshop on Multiple Access Communications (MACOM), arXiv:1411.1608 (2015)

\section{Submit your manuscript to a SpringerOpen ${ }^{\circ}$ journal and benefit from:}

- Convenient online submission

- Rigorous peer review

- Immediate publication on acceptance

- Open access: articles freely available online

- High visibility within the field

- Retaining the copyright to your article

Submit your next manuscript at $\boldsymbol{\nabla}$ springeropen.com 